\title{
Angular momentum transport in a multicomponent solar wind with differentially flowing, thermally anisotropic ions
}

\author{
B. Li and X. Li
}

Institute of Mathematics and Physics, Aberystwyth University, SY23 3BZ, UK

e-mail: [bbl;xxl]@aber.ac.uk

Received 19 September 2008 / Accepted 27 November 2008

\section{ABSTRACT}

\begin{abstract}
Context. The Helios measurements of the angular momentum flux $L$ of the fast solar wind lead to a tendency for the fluxes associated with individual ion angular momenta of protons and alpha particles, $L_{\mathrm{p}}$ and $L_{\alpha}$, to be negative (i.e., in the sense of counter-rotation with the Sun). However, the opposite holds for the slow wind, and the overall particle contribution $L_{P}=L_{\mathrm{p}}+L_{\alpha}$ tends to exceed the magnetic contribution $L_{\mathrm{M}}$. These two aspects are at variance with previous models.

Aims. We examine whether introducing realistic ion temperature anisotropies can resolve this discrepancy.

Methods. From a general set of multifluid transport equations with gyrotropic species pressure tensors, we derive the equations governing both the meridional and azimuthal dynamics of outflows from magnetized, rotating stars. The equations are not restricted to radial flows in the equatorial plane but valid for general axisymmetric winds that include two major ion species. The azimuthal dynamics are examined in detail, using the empirical meridional flow profiles for the solar wind, constructed mainly according to measurements made in situ.

Results. The angular momentum flux $L$ is determined by the requirement that the solution to the total angular momentum conservation law is unique and smooth in the vicinity of the Alfvén point, defined as where the combined Alfvénic Mach number $M_{\mathrm{T}}=1 . M_{\mathrm{T}}$ has to consider the contributions from both protons and alpha particles. Introducing realistic ion temperature anisotropies may introduce a change of up to $10 \%$ in $L$ and up to $\sim 1.8 \mathrm{~km} \mathrm{~s}^{-1}$ in azimuthal speeds of individual ions between 0.3 and 1 AU, compared with the isotropic case. The latter has strong consequences on the relative importance of $L_{P}$ and $L_{\mathrm{M}}$ in the angular momentum budget.

Conclusions. However, introducing ion temperature anisotropies cannot resolve the discrepancy between in situ measurements and model computations. For the fast-wind solutions, while in extreme cases $L_{P}$ may become negative, $L_{\mathrm{p}}$ never does. On the other hand, for the slow solar wind solutions examined, $L_{P}$ never exceeds $L_{\mathrm{M}}$, even though $L_{\mathrm{M}}$ may be less than the individual ion contribution, since $L_{\mathrm{p}}$ and $L_{\alpha}$ always have opposite signs for the slow and fast wind alike.
\end{abstract}

Key words. Sun: rotation - Sun: magnetic fields - solar wind - stars: rotation - stars: winds, outflows

\section{Introduction}

The angular momentum loss of a rotating star due to its outflow influences the rotational evolution of the star considerably, and is therefore of astrophysical significance in general (see e.g., Weber \& Davis 1967; Belcher \& MacGregor 1976; Mestel \& Spruit 1987; Bouvier et al. 1997). However, direct tests of in situ measurements against theories such as those presented by Weber \& Davis (1967) are only possible for the present Sun. A substantial number of studies have been conducted and were compiled in the comprehensive paper by Pizzo et al. (1983), who themselves paid special attention to the Helios measurements of specific angular momentum fluxes. The measurements, further analyzed by Marsch \& Richter (1984), are unique in that they allow the individual ion contribution from protons $L_{\mathrm{p}}$ and alpha particles $L_{\alpha}$ to the solar angular-momentum loss rate per steradian $L$ to be examined. For instance, despite the significant scatter, the data exhibit a distinct trend for $L_{\mathrm{p}}$ to be positive (negative) for solar winds with proton speeds $v_{\mathrm{p}}$ below (above) $400 \mathrm{~km} \mathrm{~s}^{-1}$. A similar trend for $L_{\alpha}$ is also found on average. The magnetic contribution $L_{\mathrm{M}}$, on the other hand, is remarkably constant. A mean value of $L_{\mathrm{M}}=1.6 \times 10^{29}$ dyne $\mathrm{cm} \mathrm{sr}^{-1}$ can be quoted for the solar winds of all flow speeds and throughout the region from 0.3 to $1 \mathrm{AU}$. For comparison, the mean values of angular momentum fluxes carried by ion flows in the slow solar wind are $L_{\mathrm{p}}=19.6$ and $L_{\alpha}=1.3 \times 10^{29}$ dyne $\mathrm{cm} \mathrm{sr}^{-1}$ (see Table II of Pizzo et al. 1983). The overall particle contribution to $L$ is then $L_{P}=L_{\mathrm{p}}+L_{\alpha}=20.9 \times 10^{29}$ dyne $\mathrm{cm} \mathrm{sr}^{-1}$, which tends to be larger than $L_{\mathrm{M}}$. It is noteworthy that a more recent study by Scherer et al. (2001) showed how examining the long-term variation of the non-radial components of the solar wind velocity and the corresponding angular momentum fluxes can help us understand the heliospheric magnetic field better.

Alpha particles should be placed on the same footing as protons from the perspective of solar wind modeling, given their non-negligible abundance and the fact that there tends to exist a substantial differential speed $v_{\alpha \mathrm{p}} \equiv\left|\boldsymbol{v}_{\alpha \mathrm{p}}\right| \operatorname{sign}\left(\left|\boldsymbol{v}_{\alpha}\right|-\left|\boldsymbol{v}_{\mathrm{p}}\right|\right)$. As shown by the Helios measurements, a $v_{\alpha p}$ amounting to up to $20-30 \%$ of the local proton speed may occur in both the fast and slow solar winds (Marsch et al. 1982a,b), with the latter being exemplified by an event that took place on day 117 of 1978 , when a positive $v_{\alpha \mathrm{p}} \sim 100 \mathrm{~km} \mathrm{~s}^{-1}$ was found at $0.3 \mathrm{AU}$ (Marsch et al. 1981). That on the average $v_{\alpha p} \approx 0$ in the slow wind simply reflects that the events with positive and negative $v_{\alpha p}$ occur with nearly equal frequency (Marsch et al. 1982a). As for the alpha abundance relative to protons, a value of $4.6 \%(0.4-10 \%)$ is well-established for the fast (slow) solar wind (e.g., McComas et al. 2000). Therefore alpha particles can play an important role as far as the energy and linear momentum balance of the solar wind are concerned. When it comes to the problem of angular 
momentum transport, it was shown that in interplanetary space not only the angular momentum flux carried by the alpha particles $L_{\alpha}$ but also that convected by the protons $L_{\mathrm{p}}$ are determined by the terms associated with $v_{\alpha p}(\mathrm{Li} \& \mathrm{Li} 2006)$. This essentially derives from the requirement that the proton-alpha velocity difference vector be aligned with the instantaneous magnetic field. As a consequence, these terms have no contribution to the overall angular momentum flux convected by the ion flow $L_{P}$, which turns out to be smaller than $L_{\mathrm{M}}$ in all the models examined in the parameter study by Li et al. (2007). This, together with the fact that $L_{\mathrm{p}}$ is always positive (i.e., in the sense of corotation with the Sun), is at variance with the Helios measurements.

A possible means to reconcile the measurements and the model computation is to incorporate the species temperature anisotropies. This is because the total pressure tensor $\mathrm{P}=\sum_{s} \mathrm{p}_{s}$ summed over all species $s$ participates in the problem of angular momentum transport via the component $P^{\Delta}=P^{\|}-P^{\perp}$ where $\|$ and $\perp$ are relative to the magnetic field $\boldsymbol{B}$ (see e.g., Weber 1970, hereafter referred to as W70). While the overall loss rate per steradian $L$ may not be significantly altered, the azimuthal speed of the solar wind and therefore the particle part of $L$ may be when compared with the isotropic case. Note that in the treatment of W70 the solar wind was seen as a bulk flow and the ion species are not distinguished. On the other hand, the formulation by $\mathrm{Li}$ \& Li (2006) did not take into account the pressure anisotropy, which is a salient feature of the velocity distribution functions for both protons and alpha particles as revealed by the Helios measurements (Marsch et al. 1982a,b). It therefore remains to be seen how introducing the pressure anisotropy influences individual ion azimuthal speeds. Moreover, the simple, prescribed functional form for $P^{\Delta}$ assumed in W70 needs to be updated in light of the more recent particle measurements.

The aim of the present paper is to extend the W70 study in three ways. First, we shall follow a multicomponent approach and examine the angular momentum transport in a solar wind comprising protons, alpha particles and electrons where a substantial proton-alpha particle velocity difference exists. Second, although following W70 we use a prescribed form of $P^{\Delta}$ for simplicity, this prescription is based on the Helios measurements, and also takes into account other in situ and remote sensing measurements. Third, unlike W70 where the model equations are restricted to the equatorial plane, the equation set we shall derive is appropriate for a rather general axisymmetrical, timeindependent, multicomponent, thermally anisotropic flow emanating from a magnetized rotating star. We note that a similar set of equations, which was also restricted to radial flows, was derived by Isenberg (1984) who worked in the corotating frame of reference and neglected the azimuthal dynamics altogether. The functional dependence on the radial distance and flow speed of the magnetic spiral angle was prescribed instead. His approach is certainly justifiable for the present Sun, but a self-consistent treatment of the azimuthal dynamics is required when flows from other stars are examined. This is because many stars either have a stronger magnetic field or rotate substantially faster than the Sun.

The paper is organized as follows. We start with Sect. 2 where a description is given for the general multifluid, gyrotropic transport equations, based on which the azimuthal dynamics of the multicomponent solar wind is examined. Then Sect. 3 describes the adopted meridional magnetic field and flow profiles. The numerical solutions to the angular momentum conservation law are given in Sect. 4. In Sect. 5, we shall discuss how examining the angular momentum transport in a multicomponent solar wind can also shed some light on the spectra of ion velocity fluctuations induced by Alfvénic activities. Finally,
Sect. 6 summarizes the results. The equations of and a discussion on the poloidal dynamics are presented in the Appendix.

\section{Mathematical formulation}

Presented in this section is the mathematical development of the equations that govern the angular momentum transport in a time-independent solar wind which consists of electrons (e), protons (p) and alpha particles $(\alpha)$. Each species $s(s=\mathrm{e}, \mathrm{p}, \alpha)$ is characterized by its mass $m_{s}$, electric charge $e_{s}$, number density $n_{s}$, mass density $\rho_{s}=n_{s} m_{s}$, velocity $\boldsymbol{v}_{s}$, and partial pressure tensor $\mathrm{p}_{s}$. If measured in units of the electron charge $e, e_{s}$ may be expressed by $e_{s}=Z_{s} e$ with $Z_{e} \equiv-1$ by definition.

To simplify the mathematical treatment, a number of assumptions have been made and are collected as follows:

1. Symmetry about the magnetic axis is assumed, i.e., $\partial / \partial \phi \equiv 0$ in a heliocentric spherical coordinate system $(r, \theta, \phi)$.

2. The velocity distribution function (VDF) of each species is close to a bi-Maxwellian, and the pressure tensor is gyrotropic, i.e., $\mathrm{p}_{s}=p_{s}^{\perp} \mathrm{I}+\left(p_{s}^{\|}-p_{s}^{\perp}\right) \hat{b} \hat{b}$, where $\mathrm{I}$ is the unit dyad and $\hat{b}$ is the unit vector along the magnetic field $\boldsymbol{B}$. The temperatures pertaining to the degrees of freedom parallel and perpendicular to $\boldsymbol{B}$ follow from the relation $p_{s}^{\|, \perp}=n_{s} k_{\mathrm{B}} T_{s}^{\|, \perp}$, where $k_{\mathrm{B}}$ is the Boltzmann constant.

3. Quasi-neutrality is assumed, i.e., $n_{\mathrm{e}}=\sum_{k} Z_{k} n_{k}$.

4. Quasi-zero current is assumed, i.e., $\boldsymbol{v}_{\mathrm{e}}=\sum_{k} Z_{k} n_{k} \boldsymbol{v}_{k} / n_{\mathrm{e}}(k=$ $\mathrm{p}, \alpha)$, except when the reduced meridional momentum equation is derived.

\subsection{Multi-fluid equations}

The equations appropriate for a multi-component solar wind plasma with gyrotropic species pressure tensors may be found by neglecting the electron inertia $\left(m_{\mathrm{e}} \equiv 0\right)$ in the equations given by Barakat \& Schunk (1982). Following the same procedure as given in the Appendix A.1 in Li \& Li (2006), one may find

$\nabla \cdot\left(n_{k} \boldsymbol{v}_{k}\right)=0$

$\begin{aligned} \boldsymbol{v}_{k} \cdot \nabla \boldsymbol{v}_{k} & +\frac{\nabla \cdot \mathrm{p}_{k}}{n_{k} m_{k}}+\frac{Z_{k} \nabla \cdot \mathrm{p}_{\mathrm{e}}}{n_{\mathrm{e}} m_{k}}+\frac{G M_{\odot}}{r^{2}} \hat{r} \\ & -\frac{1}{n_{k} m_{k}}\left[\frac{\delta \boldsymbol{M}_{k}}{\delta t}+\frac{Z_{k} n_{k}}{n_{\mathrm{e}}} \frac{\delta \boldsymbol{M}_{\mathrm{e}}}{\delta t}\right] \\ & -\frac{Z_{k}}{4 \pi n_{\mathrm{e}} m_{k}}(\nabla \times \boldsymbol{B}) \times \boldsymbol{B}+\frac{Z_{k} \mathrm{e}}{m_{k} c} \frac{n_{j} Z_{j}}{n_{\mathrm{e}}}\left(\boldsymbol{v}_{j}-\boldsymbol{v}_{k}\right) \times \boldsymbol{B}=0,\end{aligned}$

$\boldsymbol{v}_{s} \cdot \nabla p_{s}^{\|}+p_{s}^{\|}\left(\nabla \cdot \boldsymbol{v}_{s}+2 \nabla_{\|} \cdot \boldsymbol{v}_{s}\right)$

$$
+\nabla \cdot \boldsymbol{q}_{s}^{\|}-\mathrm{Q}_{s} \vdots \nabla(\hat{b} \hat{b})-\frac{\delta E_{s}^{\|}}{\delta t}=H_{s}^{\|},
$$

$\boldsymbol{v}_{s} \cdot \nabla p_{s}^{\perp}+p_{s}^{\perp}\left(\nabla \cdot \boldsymbol{v}_{s}+\nabla_{\perp} \cdot \boldsymbol{v}_{s}\right)$

$$
+\nabla \cdot \boldsymbol{q}_{s}^{\perp}+\frac{1}{2} \mathrm{Q}_{s}: \nabla(\hat{b} \hat{b})-\frac{\delta E_{s}^{\perp}}{\delta t}=H_{s}^{\perp},
$$

$\nabla \times\left(\boldsymbol{v}_{\mathrm{e}} \times \boldsymbol{B}\right)=0$,

where the subscript $s$ refers to all species $(s=\mathrm{e}, \mathrm{p}, \alpha)$, while $k$ stands for ion species only $(k=\mathrm{p}, \alpha)$. The gravitational constant is denoted by $G, M_{\odot}$ is the mass of the Sun, and $c$ is the speed of light. The momentum and energy exchange rates due to the Coulomb collisions of species $s$ with the remaining ones are denoted by $\delta \boldsymbol{M}_{s} / \delta t$ and $\delta E_{s}^{\|, \perp} / \delta t$, respectively. The thirdrank tensor $\mathrm{Q}_{s}$, together with the heat flux vectors $\boldsymbol{q}_{s}^{\|, \perp}$ associated with parallel and perpendicular degrees of freedom, arises 
from the deviation of species VDFs from an exact bi-Maxwellian (Barakat \& Schunk 1982). Moreover, $H_{s}^{\|, \perp}$ stands for the heating rates applied to species $s$ in the parallel and perpendicular directions from some non-thermal processes. They may be determined by assuming that the heating derives from the dissipation of Alfvén-ion cyclotron waves (e.g., Hollweg \& Isenberg 2002), or more simply in some ad hoc fashion such as employed in Leer \& Axford (1972). The operators $\nabla_{\|}$and $\nabla_{\perp}$ are defined by $\nabla_{\|}=\hat{b} \hat{b} \cdot \nabla$ and $\nabla_{\perp}=\nabla-\nabla_{\|}$, respectively.

In Eq. (2), the subscript $j$ stands for the ion species other than $k$, namely, $j=\mathrm{p}$ for $k=\alpha$ and vice versa. As can be seen, in addition to the term $(\nabla \times \boldsymbol{B}) \times \boldsymbol{B}$, the Lorentz force possesses a new term in the form of the cross product of the ion velocity difference and magnetic field. Physically, this new term represents the mutual gyration of one ion species about the other, the axis of gyration being in the direction of the instantaneous magnetic field. Furthermore, Eq. (5) is the time-independent version of the magnetic induction law, which states that the magnetic field is frozen in the electron fluid. It may be readily shown that the effects of the electron pressure gradient, the Hall term, and the momentum exchange rates as contained in the generalized Ohm's law can be safely neglected given the large spatial scale in question (a formal evaluation of the different terms can be found in Sect. 2.1 of Li et al. 2006).

To proceed, we choose a flux tube coordinate system, in which the base vectors are $\left\{\hat{e}_{l}, \hat{e}_{N}, \hat{e}_{\phi}\right\}$, where

$\hat{e}_{l}=\boldsymbol{B}_{P} /\left|\boldsymbol{B}_{P}\right|, \quad \hat{e}_{N}=\hat{e}_{\phi} \times \hat{e}_{l}$,

with the subscript $P$ denoting the poloidal component. Moreover, the independent variable $l$ is the arclength along the poloidal magnetic field line measured from its footpoint at the Sun. This choice permits the decomposition of the magnetic field and species velocities as follows,

$\boldsymbol{B}=B_{l} \hat{e}_{l}+B_{\phi} \hat{e}_{\phi}, \quad \boldsymbol{v}_{s}=v_{s l} \hat{e}_{l}+v_{s N} \hat{e}_{N}+v_{s \phi} \hat{e}_{\phi}$,

where $s=\mathrm{e}, \mathrm{p}, \alpha$. From the assumption of azimuthal symmetry, and the assumption that the solar wind is time-independent, one can see from the poloidal component of Eq. (5) that $\boldsymbol{v}_{\mathrm{e} P}$ should be strictly in the direction of $\boldsymbol{B}_{P}$. In other words, $v_{\mathrm{e} N}=0$ to a good approximation. Now let us consider the $\phi$ component of the momentum Eq. (2). Since the frequencies associated with the spatial dependence are well below the ion gyro-frequency $\Omega_{k}=\left(Z_{k} \mathrm{e} B_{l}\right) /\left(m_{k} c\right)(k=p, \alpha)$, from an order-of-magnitude estimate one can see that $\left|v_{j N}-v_{k N}\right| \ll\left|v_{k \phi}\right|$. Combined with the fact that $v_{\mathrm{e} N}=0$, this leads to that both $v_{\mathrm{p} N}$ and $v_{\alpha N}$ should be very small and can be safely neglected unless they appear alongside the ion gyro-frequency. With this in mind, one can find from the $N$ component of Eq. (2) that

$v_{\alpha \phi}-v_{\mathrm{p} \phi}=\frac{B_{\phi}}{B_{l}}\left(v_{\alpha l}-v_{\mathrm{p} l}\right)$.

That is, the ion velocity difference is strictly aligned with the magnetic field. This alignment condition further couples one ion species to the other.

The fact that $v_{s N}(s=\mathrm{e}, \mathrm{p}, \alpha)$ is negligible means that the system of vector equations may be decomposed into a force balance condition across the poloidal magnetic field and a set of transport equations along it. In the present paper, however, we simply replace the force balance condition by prescribing an analytical meridional magnetic field configuration. Moreover, we examine in detail only the azimuthal dynamics, leaving a brief discussion on the poloidal one in the Appendix.

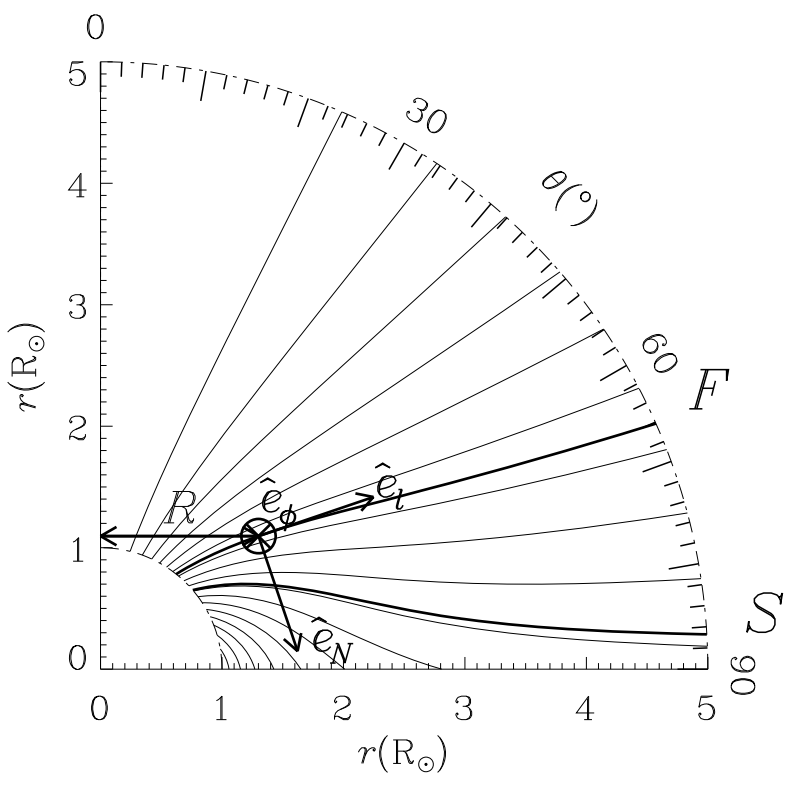

Fig. 1. Adopted meridional magnetic field configuration in the inner corona. Here only a quadrant is shown in which the magnetic axis points upward, and the thick contours labeled $F$ and $S$ delineate the lines of force along which the fast and slow solar wind solutions are examined, respectively. Also shown is how to define the geometrical factor $R$, and the base vectors $\hat{e}_{l}, \hat{e}_{N}$ and $\hat{e}_{\phi}$ of the flux tube coordinate system (see Sect. 2).

\subsection{Azimuthal dynamics}

The $\phi$ component of the magnetic induction law (5) gives

$\nabla \cdot\left[\frac{1}{R}\left(B_{\phi} \boldsymbol{v}_{\mathrm{e} P}-v_{\mathrm{e} \phi} \boldsymbol{B}_{P}\right)\right]=0$.

Now that $\boldsymbol{v}_{\mathrm{e} P}=v_{\mathrm{e}} \hat{e}_{l}$, one may readily integrate Eq. (8) along a magnetic line of force to yield

$v_{\mathrm{e} \phi}=A_{\Omega} R+\frac{B_{\phi}}{B_{l}} v_{\mathrm{e} l}$

Here $R=r \sin \theta$ is a geometrical factor to be evaluated along a given line of force (see Fig. 1), and $A_{\Omega}$ is a constant of integration and should be identified as the angular rotation rate of the footpoint of the magnetic flux tube. Taking into account the alignment condition (7), one may find that

$v_{s \phi}=A_{\Omega} R+\frac{B_{\phi}}{B_{l}} v_{s l}$

where $s=\mathrm{e}, \mathrm{p}, \alpha$. Therefore in a frame of reference that corotates with the Sun, the velocities of all species are aligned with the magnetic field.

Another equation that enters into the azimuthal dynamics is the $\phi$ component of the total momentum. In the present case, it reads

$\frac{1}{R}\left\{\sum_{k} \rho_{k} v_{k l}\left(R v_{k \phi}\right)^{\prime}-\frac{B_{l}}{4 \pi}\left[\left(1-\frac{4 \pi P^{\Delta}}{B^{2}}\right) R B_{\phi}\right]^{\prime}\right\}=0$,

where

$P^{\Delta}=P^{\|}-P^{\perp}, \quad P^{\|, \perp}=\sum_{s} p_{s}^{\|, \perp}$

and the prime' $=\hat{e}_{l} \cdot \nabla$ is the directional derivative along the poloidal magnetic field. 
For a time-independent flow $\rho_{k} v_{k l} / B_{l}=$ const. It then follows that

$R\left[v_{\mathrm{p} \phi}+\eta v_{\alpha \phi}-\frac{B_{l} B_{\phi}}{4 \pi \rho_{\mathrm{p}} v_{\mathrm{p} l}}\left(1-\frac{4 \pi P^{\Delta}}{B^{2}}\right)\right]=A_{L}$,

where the constant $\eta=\left(\rho_{\alpha} v_{\alpha l}\right) /\left(\rho_{\mathrm{p}} v_{\mathrm{p} l}\right)$ is the ion mass flux ratio, and $A_{L}$ is a constant of integration. Physically, $A_{L}$ is related to the angular momentum loss rate per steradian $L$ by

$L=\dot{M}_{\mathrm{p}} A_{L}$,

where

$\dot{M}_{k}=\rho_{k} v_{k l} \frac{B_{l \mathrm{E}}}{B_{l}} r_{\mathrm{E}}^{2}, \quad(k=\mathrm{p}, \alpha)$

is the ion mass loss rate per steradian scaled to the Earth orbit $r_{\mathrm{E}}=1 \mathrm{AU}$, with $B_{l \mathrm{E}}$ denoting the strength of the poloidal magnetic field at $r_{\mathrm{E}}$. It follows that the angular momentum loss rate of the Sun due to the solar wind $\dot{L}=4 \pi L$ if $L$ is independent of colatitude. Equation (13) shows that $L$ consists of the contributions due to individual ion angular momenta $L_{k}$, the magnetic stresses $L_{\mathrm{M}}$ and the total pressure anisotropy $L_{\text {ani }}$, where

$\left[L_{k}, L_{\mathrm{M}}, L_{\mathrm{ani}}\right]=R\left(\frac{B_{l \mathrm{E}}}{B_{l}} r_{\mathrm{E}}^{2}\right)\left[\rho_{k} v_{k l} v_{k \phi}, \frac{-B_{l} B_{\phi}}{4 \pi}, \frac{B_{l} B_{\phi} P^{\Delta}}{B^{2}}\right]$

with $k=\mathrm{p}, \alpha$.

Substituting Eqs. (10) into (13), one may find

$\tan \Phi\left[M_{\mathrm{T}}^{2}-\left(1-\beta^{\Delta} \cos ^{2} \Phi\right)\right]=\epsilon\left[\frac{A_{L}}{(1+\eta) A_{\Omega} R^{2}}-1\right]$

where $\tan \Phi=B_{\phi} / B_{l}$ defines the magnetic azimuthal angle $\Phi$, and

$M_{\mathrm{T}}^{2}=M_{\mathrm{p}}^{2}+M_{\alpha}^{2}, M_{k}^{2}=\frac{4 \pi \rho_{k} v_{k l}^{2}}{B_{l}^{2}}(k=\mathrm{p}, \alpha)$,

$\beta^{\Delta}=\frac{4 \pi P^{\Delta}}{B_{l}^{2}}, \epsilon=(1+\eta) M_{\mathrm{p}}^{2} \frac{A_{\Omega} R}{v_{\mathrm{p} l}}$.

By definition, $M_{\mathrm{T}}$ is the combined poloidal Alfvénic Mach number, which involves both ion species. For a typical solar wind, between $1 R_{\odot}$ and $1 \mathrm{AU}$ there exists a point where $M_{\mathrm{T}}=1$, which is to be called the Alfvén point and denoted by $r_{\mathrm{a}}$.

As discussed in detail by Li \& Li (2006), when species temperature anisotropy is absent $\left(P^{\Delta}=0\right.$ and therefore $\left.\beta^{\Delta}=0\right)$, for Eq. (17) to possess a solution that passes smoothly through $r_{\mathrm{a}}$ the two constants $A_{L}$ and $A_{\Omega}$ have to be related by

$A_{L}=(1+\eta) A_{\Omega} R_{\mathrm{a}}^{2}$,

where the subscript $a$ denotes quantities evaluated at the Alfvén point. When $\beta^{\Delta}$ is not zero, a direct relation between $A_{L}$ and $A_{\Omega}$ is not as obvious since now Eq. (17) becomes cubic in $\tan \Phi$. Nevertheless, one may write $A_{L}$ as $A_{L}=\lambda A_{L 0}$, where $A_{L 0}$ is determined through Eq. (19) and therefore $\lambda$ stands for the correction due to a finite $\beta^{\Delta}$. It then follows that

$c_{3} \tan ^{3} \Phi+c_{2} \tan ^{2} \Phi+c_{1} \tan \Phi+c_{2}=0$,

where

$c_{3}=M_{\mathrm{T}}^{2}-1, c_{2}=\epsilon\left[1-\lambda\left(\frac{R_{\mathrm{a}}}{R}\right)^{2}\right]$,

$c_{1}=M_{\mathrm{T}}^{2}-1+\beta^{\Delta}$.
Given the meridional flow profiles along a prescribed magnetic field line, Eq. (20) possesses only one real root at locations far away from $r_{\mathrm{a}}$. However, in the vicinity of $r_{\mathrm{a}}$, there exists in general three real roots and they diverge near $r_{\mathrm{a}}$. The requirement that there exists a unique solution that is smooth from $1 R_{\odot}$ out to 1 AU determines $\lambda$ (Weber \& Davis 1970; Weber 1970).

\section{Meridional magnetic field and flow profiles}

In principle, one needs to solve Eqs. (A.1) to (A.4) together with Eq. (17) simultaneously to gain a a quantitative insight. In the present paper, we refrain from doing so because from previous experience it proves difficult to yield the flow profiles that satisfactorily reproduce in situ measurements such as made by Helios. Take the proton-alpha speed difference $v_{\alpha p}$ in the fast solar wind for example. It is observationally established that $v_{\alpha \mathrm{p}}$ closely tracks the local Alfvén speed in the heliocentric range $r>0.3$ AU (Marsch et al. 1982a). So far this fact still poses a theoretical challenge: adjusting the ad hoc heating parameters, or fine-tuning the cyclotron resonance mechanism is unable to produce such a behavior (see, e.g. Hu \& Habbal 1999). We therefore adopt an alternative approach by prescribing the background meridional flow profiles that mimic the observations and then examining what consequences the species anisotropies have on the azimuthal dynamics.

\subsection{Background meridional magnetic field}

For the meridional magnetic field, we adopt an analytical model given by Banaszkiewicz et al. (1998). In the present implementation, the model magnetic field consists of the dipole and currentsheet components only. A set of parameters $M=2.2265, Q=0$, $K=0.9343$ and $a_{1}=1.5$ are chosen such that the last open magnetic field line is anchored at heliocentric colatitude $\theta=50^{\circ}$ on the Sun, while at the Earth orbit, the meridional magnetic field strength $B_{l}$ is $3 \gamma$ and independent of colatitude $\theta$, consistent with Ulysses measurements (Smith \& Balogh 1995).

The background magnetic field configuration is depicted in Fig. 1, where the thick contours labeled $F$ and $S$ represent the lines of force along which we examine the fast and slow solar wind solutions, respectively. Tube $F(S)$, which intersects the Earth orbit at $70^{\circ}\left(89^{\circ}\right)$ colatitude, originates from $\theta=38.5^{\circ}\left(49.4^{\circ}\right)$ at the Sun where the meridional magnetic field strength $B_{l}$ is $3.93(3.49) \mathrm{G}$.

\subsection{Prescribed meridional flow profiles}

The background meridional flow parameters are found by adopting a three-step approach described as follows:

1. Using some ad hoc heating parameters, we solve along flux tube $F(S)$ the isotropic version of Eqs. (A.1) to (A.4) (see Eqs. (8) to (10) in $\mathrm{Li} \& \mathrm{Li} 2007$, for details) to yield the distribution between $1 R_{\odot}$ and $1 \mathrm{AU}$ of the ion number densities $n_{k}$ and meridional speeds $v_{k l}(k=p, \alpha)$, as well as the isotropic species temperatures $T_{s}(s=\mathrm{e}, \mathrm{p}, \alpha)$ for the fast (slow) solar wind. Specifically, the heating rates are of the same format as in Sect. 3.2 in Li \& Li (2008). To generate the fast and slow solar wind solutions, the parameters $\left[F_{\mathrm{E}}\right.$ (in erg $\mathrm{cm}^{-2} \mathrm{~s}^{-1}$ ), $l_{d}$ (in $R_{\odot}$ ), $\chi$ ] are chosen to be $[1.9,2.2,2.2]$ and $[1,1.8,3.7]$, respectively. By simply adjusting the heating parameters it proves difficult to produce a reasonable $T_{\mathrm{p}}$ profile in that if $T_{\mathrm{p}}$ in the inner corona is close to observations then $T_{\mathrm{p}}$ at $1 \mathrm{AU}$ is usually only a fraction of the 
typically measured values. Moreover, the derived speed difference $v_{\alpha \mathrm{p}, l}=v_{\alpha l}-v_{\mathrm{p} l}$ varies little between 0.3 and $1 \mathrm{AU}$, in contrast to the Helios measurements. Therefore some additional steps are employed to make the flow profiles more realistic. Specifically, all the parameters except $n_{\mathrm{p}}$ and $v_{\mathrm{p} l}$ are required to undergo a smooth transition from the profiles for the region $r \lessgtr 0.3 \mathrm{AU}$ derived so far to those specified in next step for the outer region.

2. The desired profiles, given in Table 1 , for $v_{\alpha \mathrm{p}, l}, T_{\mathrm{p}}$ and $T_{\alpha}$ for the region $r \gtrsim 0.3 \mathrm{AU}$ (denoted by subscript $o$ ) are based on the in situ measurements to be detailed shortly. Once $v_{\alpha \mathrm{p}, l}$ is known, the meridional alpha speed $v_{\alpha l}$ is given by $v_{\mathrm{p} l}+$ $v_{\alpha \mathrm{p}, l}$, and the alpha density $n_{\alpha}$ by $\left(n_{\alpha}\right)_{I}\left(v_{\alpha l}\right)_{I} / v_{\alpha l}$, where the subscript $I$ denotes the values obtained in the first step. The distributions of $n_{k}, v_{k l}(k=\mathrm{p}, \alpha)$ and $T_{s}(s=\mathrm{e}, \mathrm{p}, \alpha)$ thus constructed are for the isotropic model.

3. Now the ion temperatures $T_{k}^{\|, \perp}$ can be constructed by prescribing the temperature anisotropy $\Gamma_{k}=T_{k}^{\|} / T_{k}^{\perp}(k=\mathrm{p}, \alpha)$. Note that the electron temperature is assumed to be isotropic. For the region within several solar radii, $\Gamma_{k}$ is required to decrease with $r$ from 1 at $1 R_{\odot}$, where the Coulomb selfcollisions are still frequent enough to suppress a temperature anisotropy, to some value less than unity. This inner profile is not directly constrained by observations but constructed by noting that the processes operational in the inner corona tend to heat the ions preferentially in the perpendicular direction (e.g., Hollweg \& Isenberg 2002). On the other hand, for $r \gtrsim 0.3 \mathrm{AU}, \Gamma_{k}$ follows a power law dependence on $r$ with the exponent determined by the Helios measurements (see Table 1). Specifying the temperature anisotropies of protons and alpha particles at $1 \mathrm{AU}, \Gamma_{\mathrm{pE}}$ and $\Gamma_{\alpha \mathrm{E}}$, determines $\Gamma_{k}$ in the outer region. The inner and outer profiles are then connected smoothly to yield the desired $\Gamma_{k}$. The temperatures $T_{k}^{\|, \perp}$ follow from the relations $T_{k}^{\perp}=3 T_{k} /\left(2+\Gamma_{k}\right)$ and $T_{k}^{\|}=\Gamma_{k} T_{k}^{\perp}$.

A detailed description of Table 1 is necessary. Note that throughout this table $x=r / r_{\mathrm{E}}$ where $r_{\mathrm{E}}=1$ AU. Let us first focus on the adopted values for the fast solar wind. For the isotropic proton and alpha temperatures at $1 \mathrm{AU}$, we adopted the typical values of $T_{\mathrm{p}}=2.8 \times 10^{5} \mathrm{~K}$ and $T_{\alpha}=5 T_{\mathrm{p}}$ (see e.g., Schwenn 1990; McComas et al. 2000, hereafter Sch90 and Mc00). Furthermore, Figs. 18 and 19 in Marsch et al. (1982b, hereafter M82b) indicate that $T_{\mathrm{p}}^{\|} \propto x^{-0.75}$, and $T_{\mathrm{p}}^{\perp} \propto x^{-1.08}$. A power law dependence for $T_{\mathrm{p}}$ of $T_{\mathrm{p}} \propto x^{-1}$ is therefore consistent with such a behavior, and also consistent with the Ulysses measurements (see Table 2 in Mc00). Furthermore, Fig. 5 in Marsch et al. (1982a, hereafter M82a) indicates that $T_{\alpha}^{\|} \propto x^{-1.15}$, and $T_{\alpha}^{\perp} \propto x^{-1.38}$. A profile of $T_{\alpha} \propto x^{-1.3}$ is consistent with this behavior, but differs substantially from that measured by Ulysses, which yields that $T_{\alpha} \propto x^{-0.8}$ (see Table 2 in Mc00). Moving on to the slow solar wind, we note that values of $T_{\mathrm{p}}=5.5 \times 10^{4} \mathrm{~K}$ and $T_{\alpha}=1.7 \times$ $10^{5} \mathrm{~K}$ are typically found at $1 \mathrm{AU}$ (see e.g., Sch90). In addition, Figs. 18 and 19 in M82b indicate that $T_{\mathrm{p}}^{\|} \propto x^{-1.03}$, and $T_{\mathrm{p}}^{\perp} \propto x^{-0.9}$. Therefore we adopted a $T_{\mathrm{p}}$ profile of $T_{\mathrm{p}} \propto x^{-0.94}$. On the other hand, we adopted a profile for $T_{\alpha}$ in the form $T_{\alpha} \propto$ $x^{-0.96}$, which is consistent with the measured alpha temperature anisotropies which indicate that $T_{\alpha}^{\|} \propto x^{-0.83}$, and $T_{\alpha}^{\perp} \propto x^{-1.02}$ (see Fig. 5 in M82a).

In this study $\Gamma_{\mathrm{pE}}$ and $\Gamma_{\alpha \mathrm{E}}$ will serve as free parameters. The Helios measurements indicate that $\Gamma_{\mathrm{pE}} \approx 1.2 \pm 0.3$ and $\Gamma_{\alpha \mathrm{E}} \approx$ $1.3 \pm 0.6$ for the fast solar wind with $v_{\mathrm{pl}} \gtrsim 600 \mathrm{~km} \mathrm{~s}^{-1}$, while $\Gamma_{\mathrm{pE}} \approx 1.7 \pm 0.7$ and $\Gamma_{\alpha \mathrm{E}} \approx 1.4 \pm 0.6$ for the slow solar wind with $v_{\mathrm{p} l} \lesssim 400 \mathrm{~km} \mathrm{~s}^{-1}$ (Marsch et al. 1982a,b). Theoretically, one may expect that the $\left[\Gamma_{\mathrm{pE}}, \Gamma_{\alpha \mathrm{E}}\right]$ pair may not occupy the whole rectangle bounded by the given values in the $\Gamma_{\mathrm{pE}}-\Gamma_{\alpha \mathrm{E}}$ space, since too strong an anisotropy can drive the system unstable with respect to a number of instabilities when the plasma $\beta$ is comparable to unity. Given that the lower limit of $\Gamma_{\mathrm{pE}}$ or $\Gamma_{\alpha \mathrm{E}}$ is only slightly lower than 1, the ion-cyclotron instability can be shown to be unlikely to occur (see, e.g., Eq. (3) in Gary et al. 1994). However, the firehose instability may be relevant since it happens when $P^{\|}$ is sufficiently larger than $P^{\perp}$ and $\beta^{\|}=8 \pi P^{\|} / B^{2} \gtrsim 1$. Note that the alpha particles with a non-negligible abundance drifting relative to protons may complicate the situation considerably given that in addition to the firehose, electromagnetic ion/ion instabilities may also be relevant and the occurrence of such instabilities is not restricted to the cases where the parallel $\beta$ is large (Hellinger \& Trávníček 2006). Nevertheless, we only compare the modeled $\left[\Gamma_{\mathrm{p}}, \Gamma_{\alpha}\right]$ with the non-resonant firehose criterion such as found via the dispersion relation of Alfvén waves (see Eq. (23) in Isenberg 1984). Specializing to an electron-protonalpha plasma, the dispersion relation dictates that instability occurs when $1-P^{\perp} / P^{\|}>2\left(1-x_{\mathrm{p}} x_{\alpha}\right) / \beta^{\|}$where $x_{k}=\left(\rho_{k} / \rho\right)\left(v_{\alpha \mathrm{p}} / v_{\mathrm{A}}\right)$ $(k=\mathrm{p}, \alpha)$ with $v_{\mathrm{A}}=B / \sqrt{4 \pi \rho}$ being the Alfvén speed determined by the bulk mass density $\rho=\rho_{\mathrm{p}}+\rho_{\alpha}$. Using this criterion it is found that the modeled flow profiles are all stable with the only exception being for the segment $r \gtrsim 195 R_{\odot}$ in the fast wind with the largest values of $\Gamma_{\mathrm{pE}}$ and $\Gamma_{\alpha \mathrm{E}}$.

Figure 2 gives the radial distributions between $1 R_{\odot}$ and $1 \mathrm{AU}$ of the flow parameters for the fast and slow solar wind in the left and right panels, respectively. Figures $2 \mathrm{a}$ and $\mathrm{c}$ depict the meridional ion speeds $v_{\mathrm{p} l}$ and $v_{\alpha l}$, while the ion temperatures $T_{k}^{\|}$(the dotted curves), $T_{k}^{\perp}$ (dashed) and $T_{k}$ (solid) are given in Figs. $2 \mathrm{~b}$ and $\mathrm{d}(k=\mathrm{p}, \alpha)$. The values for the temperature anisotropy adopted for the construction are $\Gamma_{\mathrm{p}, \mathrm{E}}=1.5$ and $\Gamma_{\alpha, \mathrm{E}}=1.9$ for the fast wind, and $\Gamma_{\mathrm{p}, \mathrm{E}}=2.42$ and $\Gamma_{\alpha, \mathrm{E}}=2$ for the slow wind. In Fig. $2 b$, the error bars represent the uncertainties of the UVCS measurements for the proton effective temperature, made for a polar coronal hole as reported by Kohl et al. (1998). Similar measurements by Frazin et al. (2003) along the edges of an equatorial streamer are given in Fig. 2d. Moreover, the asterisks in Figs. 2a and c mark the location of the Alfvén point as defined by Eq. (18).

For the fast (slow) solar wind it is found that at $1 \mathrm{AU}$ the meridional proton speed $v_{\mathrm{p} l}$ is $607(304) \mathrm{km} \mathrm{s}^{-1}$, the proton flux $n_{\mathrm{p}} v_{\mathrm{p} l}$ is 2.8 (3.84) in units of $10^{8} \mathrm{~cm} \mathrm{~s}^{-2}$, the alpha abundance $n_{\alpha} / n_{\mathrm{p}}$ is $4.56 \%$ (3.6\%), and the meridional component of the proton-alpha velocity difference $v_{\alpha \mathrm{p}, l}$ is $23(5) \mathrm{km} \mathrm{s}^{-1}$. These values are consistent with in situ measurements such as made by Ulysses (McComas et al. 2000). Moreover, the fast (slow) solar wind reaches the Alfvén point at 10.7 (13.3) $R_{\odot}$, beyond which $v_{\mathrm{p} l}$ increases only slightly with increasing $r$. On the other hand, for $r \gtrsim 0.3 \mathrm{AU}$ the meridional alpha speed $v_{\alpha l}$ decreases rather than increases with $r$ as a consequence of the prescribed $v_{\alpha \mathrm{p}, l}$ profile. If examining the ratio of $v_{\alpha \mathrm{p}, l}$ to the meridional Alfvén speed $v_{\mathrm{A} l}=B_{l} / \sqrt{4 \pi \rho}$, one may find that for the fast solar wind this ratio decreases only slightly from 0.98 at $0.3 \mathrm{AU}$ to 0.82 at $1 \mathrm{AU}$, while for the slow wind it shows a substantial variation from 0.88 at $0.3 \mathrm{AU}$ to 0.29 at $1 \mathrm{AU}$. The modeled $v_{\alpha \mathrm{p}, l} / v_{\mathrm{A} l}$ can be seen to agree with the Helios measurements as given by Fig. 11 of Marsch et al. (1982a). Note that a value of $v_{\alpha \mathrm{p}, l}=49 \mathrm{~km} \mathrm{~s}^{-1}$ at $0.3 \mathrm{AU}$ is not unrealistic for slow solar winds, even larger values have been found by Helios 2 when approaching perihelion (Marsch et al. 1981). Moving on to the temperature profiles, one may see that the $T_{\mathrm{p}}^{\perp}$ profiles inside $5 R_{\odot}$ are in reasonable 

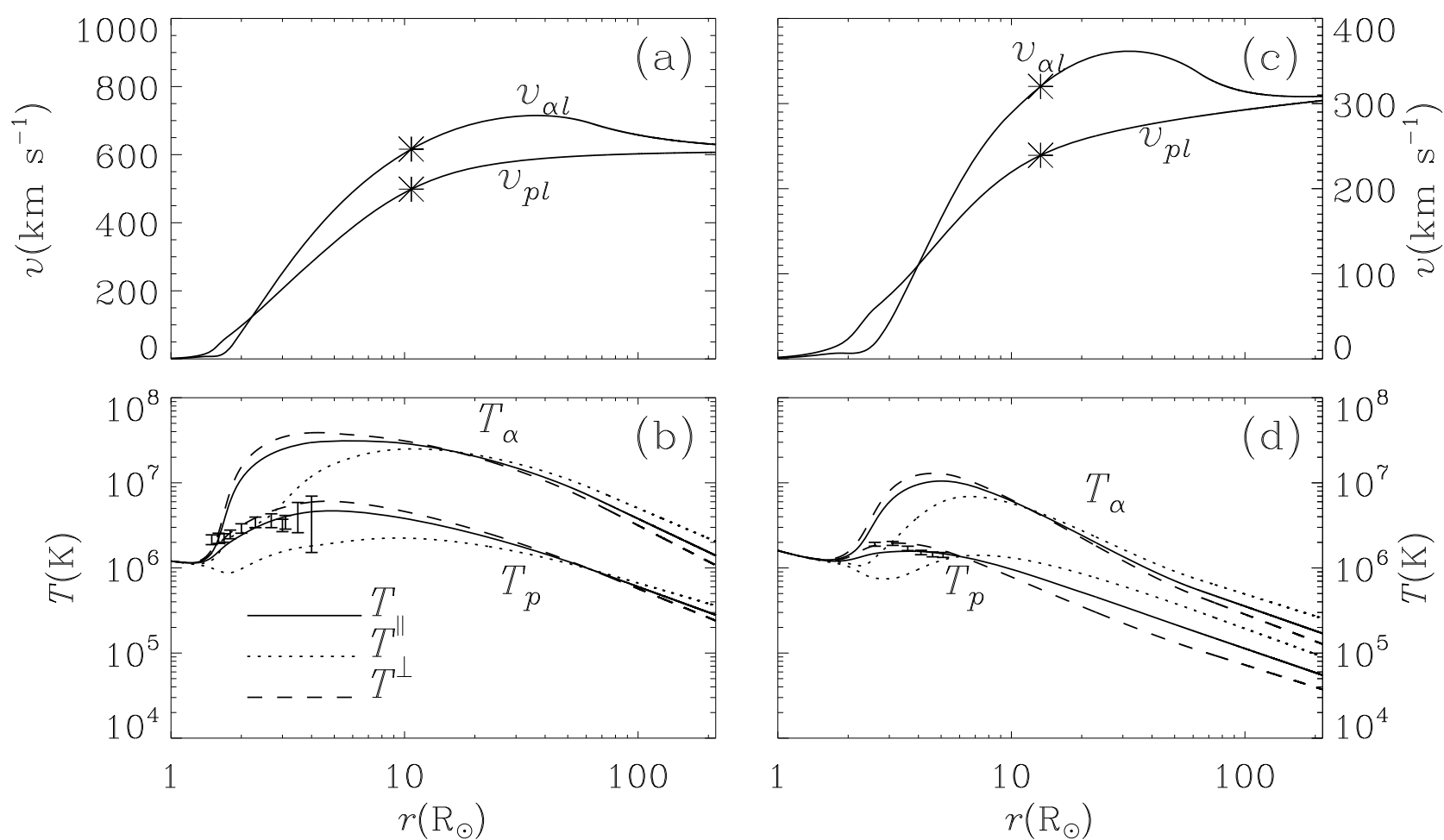

Fig. 2. Radial distribution between $1 R_{\odot}$ and $1 \mathrm{AU}$ of the adopted meridional flow parameters for the fast (left column) and slow (right) solar wind. a) and $\mathbf{c}$ ), the meridional flow speeds of protons $\left(v_{\mathrm{pl}}\right)$ and alpha particles $\left(v_{\alpha l}\right)$. b) and $\mathbf{d}$ ), the ion temperatures $T_{k}^{\|}$(dotted lines), $T_{k}^{\perp}$ (dashed lines), and $T_{k}=\left(T_{k}^{\|}+2 T_{k}^{\perp}\right) / 3$ (solid lines) where $k=\mathrm{p}, \alpha$. The construction of $T_{k}^{\|, \perp}$ is described in Sect. 3.2. The error bars in b) and $\mathbf{d}$ ) represent the uncertainties of the UVCS measurements of the effective proton temperature as reported by Kohl et al. (1998) for a coronal hole, and by Frazin et al. (2003) for a streamer, respectively. Note that both measurements are typical of solar minimum conditions. Moreover, the asterisks in a) and c) denote the Alfvén point, where the meridional Alfvénic Mach number (defined by Eq. (18)) equals unity.

agreement with the UVCS line-width measurements for both the fast and slow solar wind.

\section{Numerical results}

Having described the meridional magnetic field and flow profiles, we may now address the following questions: to what extent is the total angular momentum loss of the Sun affected by the ion temperature anisotropies? and how is the angular momentum budget distributed among particle momenta, the magnetic torque, and the torque due to ion temperature anisotropies? To this end, let us first examine the fast and then the slow solar wind solutions. In the computations, we take $A_{\Omega}=2.865 \times$ $10^{-6} \mathrm{rad} \mathrm{s}^{-1}$, which corresponds to a sidereal rotation period of 25.38 days.

\subsection{Fast solar wind}

Figure 3 presents the radial profiles of (a) the proton azimuthal speed $v_{\mathrm{p} \phi} ;$ (b) the alpha one $v_{\alpha \phi}$; and (c) the ion angular momentum fluxes $L_{k}(k=\mathrm{p}, \alpha)$, their sum $L_{P}$, the flux due to the magnetic torque $L_{\mathrm{M}}$, and that due to temperature anisotropies $L_{\text {ani }}$ (see Eq. (16)). Note that the dash-dotted curves in Fig. 3c plot negative values. In Figs. $3 a$ and $b$, the ion azimuthal speeds for the isotropic model with identical meridional flow parameters are given by the dashed lines for comparison. The fast wind profile corresponds to $\Gamma_{\mathrm{pE}}=1.5$ and $\Gamma_{\alpha \mathrm{E}}=1.9$.

For the chosen $\Gamma_{\mathrm{pE}}$ and $\Gamma_{\alpha \mathrm{E}}$, it is found that $\lambda=1.058$. Consequently, the total angular momentum loss rate per steradian $L$ is 1.8 (here and hereafter in units of $10^{29}$ dyne $\mathrm{cm} \mathrm{sr}^{-1}$ ) in the anisotropic case, and is only modestly enhanced compared with the isotropic case, for which $L=1.71$. Furthermore, Figs. $3 \mathrm{a}$ and $\mathrm{b}$ indicate that the radial dependence of the ion azimuthal speed $v_{\mathrm{p} \phi}$ or $v_{\alpha \phi}$ in the anisotropic model is similar to that in the isotropic one. For instance, both models yield that with increasing distance the alpha particles develop an azimuthal speed in the direction of counterrotation with the Sun: $v_{\alpha \phi}$ becomes negative beyond 7.95 (8.35) $R_{\odot}$ in the anisotropic (isotropic) model. The difference between the isotropic and anisotropic cases becomes more prominent at large distances where $\beta^{\Delta}$ becomes increasingly significant, as would be expected from Eq. (17). Take the values of $v_{\mathrm{p} \phi}$ and $v_{\alpha \phi}$ at $1 \mathrm{AU}$. The isotropic (anisotropic) model yields that $v_{\mathrm{p} \phi}=2.54(3.46) \mathrm{km} \mathrm{s}^{-1}$ and that $v_{\alpha \phi}=-12.6(-11.7) \mathrm{km} \mathrm{s}^{-1}$ at 1 AU. Note that the changes introduced to the ion azimuthal speeds by pressure anisotropies $\left(\sim 0.9 \mathrm{~km} \mathrm{~s}^{-1}\right.$ for both protons and alpha particles) play an important role in the distribution of the angular momentum budget $L$ among different contributions, as shown by Fig. 3c. The proton contribution $L_{\mathrm{p}}$ exceeds $L_{\mathrm{M}}$ for $r \gtrsim 57 R_{\odot}$ and $L_{\mathrm{p}}$ attains 5.07 at $1 \mathrm{AU}$, significantly larger than the magnetic part $L_{\mathrm{M}}=1.48$ at the same location. In fact, the overall particle contribution $L_{P}$, which increases with distance, overtakes the magnetic contribution $L_{\mathrm{M}}$ from $170.5 R_{\odot}$ onwards, despite the fact that the alpha contribution tends to offset the proton one. The dominance of $L_{P}$ over $L_{\mathrm{M}}$ happens in conjunction with the increasing importance of $L_{\text {ani }}$, the flux due to total pressure anisotropy which is in the direction of counterrotation with the Sun. In contrast, without pressure anisotropies, at $1 \mathrm{AU}$ it turns out that even though a value of 3.73 is found for $L_{\mathrm{p}}$, it is almost cancelled by an $L_{\alpha}$ of -3.49 . The resulting $L_{P}$ is thus 0.23 , substantially smaller than 
Table 1. Profiles for some solar wind parameters in the region $r>$ $0.3 \mathrm{AU}$.

\begin{tabular}{lcc}
\hline \hline & Fast wind & Slow wind \\
\hline$\left(v_{\alpha \mathrm{p}, l}\right)_{o}$ & $23 x^{-1.15} \mathrm{~km} \mathrm{~s}^{-1 a}$ & $5 x^{-1.9} \mathrm{~km} \mathrm{~s}^{-1}$ \\
$\left(T_{\mathrm{p}}\right)_{o}$ & $2.8 \times 10^{5} x^{-1} \mathrm{~K}$ & $5.5 \times 10^{4} x^{-0.94} \mathrm{~K}$ \\
$\left(T_{\alpha}\right)_{o}$ & $1.4 \times 10^{6} x^{-1.3} \mathrm{~K}$ & $1.7 \times 10^{5} x^{-0.96} \mathrm{~K}$ \\
$\left(\Gamma_{\mathrm{p}}\right)_{o}$ & $\Gamma_{\mathrm{pE}} x^{0.33}$ & $\Gamma_{\mathrm{pE}} x^{-0.13}$ \\
$\left(\Gamma_{\alpha}\right)_{o}$ & $\Gamma_{\alpha \mathrm{E}} x^{0.23}$ & $\Gamma_{\alpha \mathrm{E}} x^{0.19}$ \\
\hline
\end{tabular}

${ }^{a}$ Please see Sect. 3.2 for details.

$L_{\mathrm{M}}$, which is nearly identical to the value found in the anisotropic model. This contrast between anisotropic and isotropic cases is understandable since it follows from Eq. (13) that, given that the constant $A_{L}$ does not vary much from the isotropic to anisotropic model, the change of $L_{P}$ should be largely offset by that of $L_{\text {ani }}$.

Figure 4 expands the obtained results by displaying the dependence on $\Gamma_{\mathrm{pE}}$ and $\Gamma_{\alpha \mathrm{E}}$ of (a) the factor $\lambda$, (b) the proton azimuthal speed $v_{\mathrm{p} \phi}$ and (c) the alpha one $v_{\alpha \phi}$ at two different distances plotted by the different linestyles indicated in (b), as well as (d) the constituents comprising the angular momentum flux at $1 \mathrm{AU}$. In addition to the individual ion contributions $L_{\mathrm{p}}$ and $L_{\alpha}$, and the magnetic one $L_{\mathrm{M}}$, the overall particle contribution $L_{P}=L_{\mathrm{p}}+L_{\alpha}$ is also given. Note that $-L_{\alpha}$ instead of $L_{\alpha}$ is plotted in Fig. 4d. Moreover, the horizontal bars on the left of Figs. $4 b$ and $\mathrm{c}$ represent the azimuthal ion speeds derived in the isotropic case at the corresponding locations for comparison. The open circles correspond to the cases where $\Gamma_{\alpha \mathrm{E}}=1.3$. It turns out that at any given $\Gamma_{\mathrm{pE}}$ each parameter varies monotonically from the value with $\Gamma_{\alpha \mathrm{E}}=0.7$, represented by the end of the arrow, to the value with $\Gamma_{\alpha \mathrm{E}}=1.9$ given by the arrow head. In Fig. $4 \mathrm{~b}$ the arrows have been slightly shifted from one another to avoid overlapping.

From Fig. 4a one can see that $\lambda$ decreases with increasing $\Gamma_{\mathrm{pE}}$ or $\Gamma_{\alpha \mathrm{E}}$, ranging from 1.101 at the upper left to 1.058 at the lower right corner. The deviation of $\lambda$ from unity, albeit modest, indicates that the changes introduced in the total angular momentum loss due to the ion pressure anisotropies are not negligible. From Figs. $4 \mathrm{~b}$ and $\mathrm{c}$ one can see that between 0.3 to $1 \mathrm{AU}$, the magnitude of the azimuthal speeds of both species decreases with increasing distance. Furthermore, at either 0.3 or $1 \mathrm{AU}$, both $v_{\mathrm{p} \phi}$ and $v_{\alpha \phi}$ increase when $\Gamma_{\mathrm{pE}}$ or $\Gamma_{\alpha \mathrm{E}}$ increases. Take the values at $1 \mathrm{AU}$ for instance. One can see that $v_{\mathrm{p} \phi}$ ranges from 2.36 to $3.46 \mathrm{~km} \mathrm{~s}^{-1}$, while $v_{\alpha \phi}$ varies between -12.8 and $-11.7 \mathrm{~km} \mathrm{~s}^{-1}$. For the majority of the solutions both $v_{\mathrm{p} \phi}$ and $v_{\alpha \phi}$ tend to be larger in the algebraic sense than the corresponding values in the isotropic model, which yield 2.54 and $-12.6 \mathrm{~km} \mathrm{~s}^{-1}$ for protons and alpha particles, respectively. However, at $0.3 \mathrm{AU}$ $v_{\mathrm{p} \phi}$ or $v_{\alpha \phi}$ tends to be smaller in the anisotropic than in the isotropic case. Now $v_{\mathrm{p} \phi}$ and $v_{\alpha \phi}$ vary in the intervals [2.37, 3.77] and $[-15.8,-14.2] \mathrm{km} \mathrm{s}^{-1}$, respectively. For comparison, the isotropic model yields a $v_{\mathrm{p} \phi}\left(v_{\alpha \phi}\right)$ of $3.33(-14.7) \mathrm{km} \mathrm{s}^{-1}$. Now let us examine the specific angular momentum fluxes $L_{\mathrm{p}}, L_{\alpha}$ and $L_{\mathrm{M}}$ at $1 \mathrm{AU}$. Figure $4 \mathrm{~d}$ indicates that $L_{\mathrm{M}}$ has the weakest parameter dependence, which is easily understandable given that to a good approximation $\tan \Phi \approx-A_{\Omega} R / v_{k l}$ where $k$ may be taken to be p or $\alpha$ (see Eq. (10)). Besides, the parameter dependence of $L_{\alpha}$ is rather modest, varying by $\lesssim 10 \%$ from -3.55 to -3.23 when $\Gamma_{\mathrm{pE}}$ or $\Gamma_{\alpha \mathrm{E}}$ changes. On the other hand, $L_{\mathrm{p}}$ changes substantially, ranging between 3.46 and 5.07. Hence the overall particle contribution $L_{P}$ also shows a significant parameter dependence. In particular, $L_{P}$ may exceed $L_{\mathrm{M}}$ when $\Gamma_{\mathrm{pE}} \gtrsim 1.3$. For the solutions examined, $L_{P}$ can be found to be positive and attain
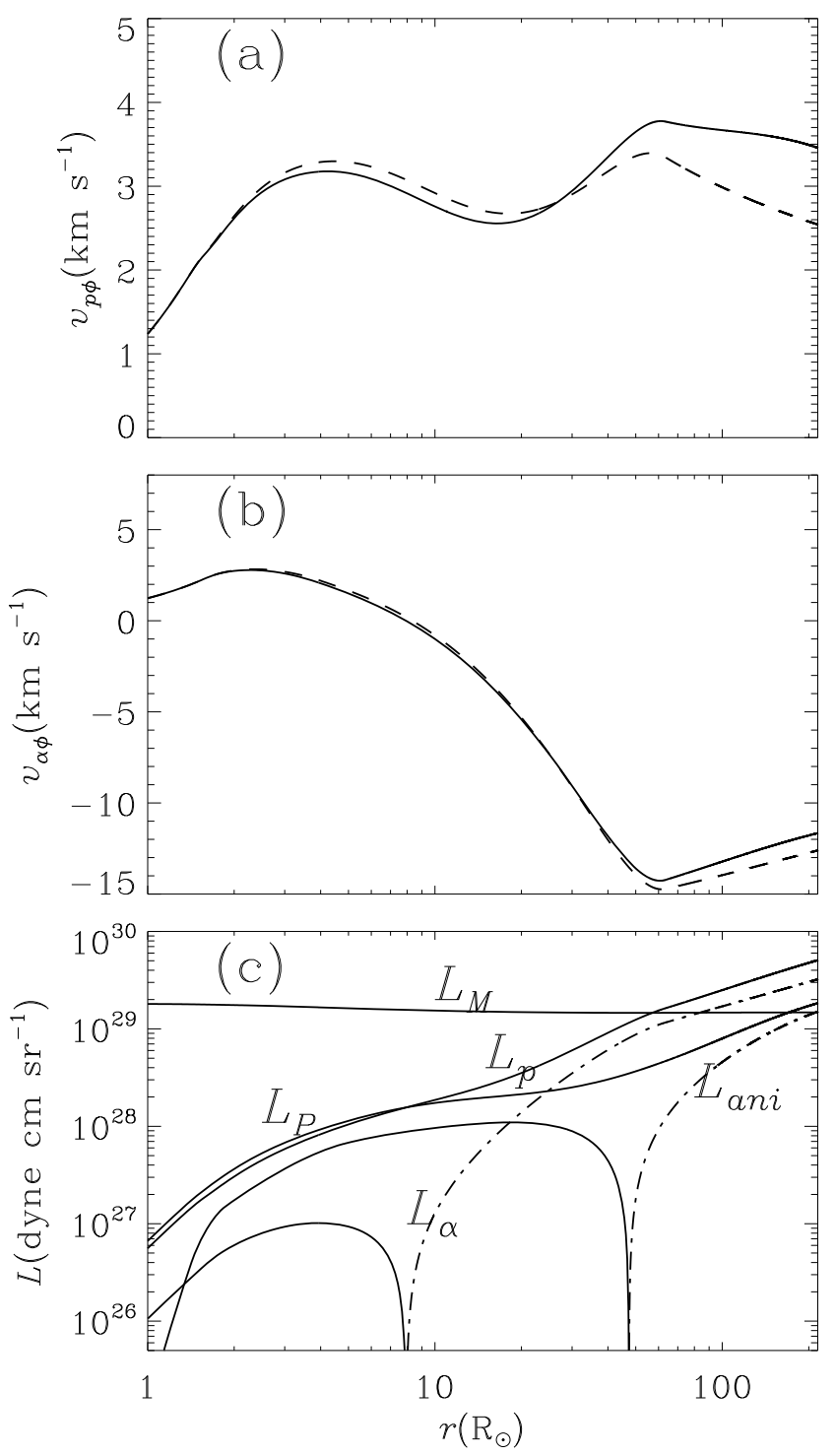

Fig. 3. Radial distributions of a) the proton azimuthal speed $v_{\mathrm{p} \phi}$, b) the alpha one $v_{\alpha \phi}$, and c) various contributions to the angular momentum budget in an $\mathrm{e}-\mathrm{p}-\alpha$ solar wind with ion temperature anisotropies. In a) and b), the profiles derived for a solar wind with identical flow parameters where ion temperature anisotropies are neglected are given by dashed lines for comparison. Panel c) depicts the individual ion angular momentum fluxes $L_{\mathrm{p}}$ and $L_{\alpha}$, their sum $L_{P}$, and the fluxes associated with the magnetic stresses $L_{\mathrm{M}}$, and with the temperature anisotropies $L_{\text {ani }}$ (see Eq. (16)). The dash-dotted lines represent negative values.

its maximum of $L_{P}=1.84$ when $\left[\Gamma_{\mathrm{pE}}, \Gamma_{\alpha \mathrm{E}}\right]=[1.5,1.9]$. Only for the lowest values of $\Gamma_{\mathrm{pE}}$ and $\Gamma_{\alpha \mathrm{E}}$ can one find a negative $L_{P}$ of -0.084 . Moreover, the protons always show a partial corotation, i.e., $L_{\mathrm{p}}>0$. From this we conclude that the ion temperature anisotropies are unlikely the cause of the tendency for $L_{\mathrm{p}}$ or $L_{P}$ to be negative for the fast solar wind as indicated by the Helios measurements (Pizzo et al. 1983; Marsch \& Richter 1984).

\subsection{Slow solar wind}

Figure 5 presents, in the same fashion as Fig. 4, the dependence on $\Gamma_{\mathrm{pE}}$ and $\Gamma_{\alpha \mathrm{E}}$ of various quantities derived for the slow solar wind. A comparison with Fig. 4 indicates that nearly all the features in Fig. 5 are reminiscent of those obtained for fast 


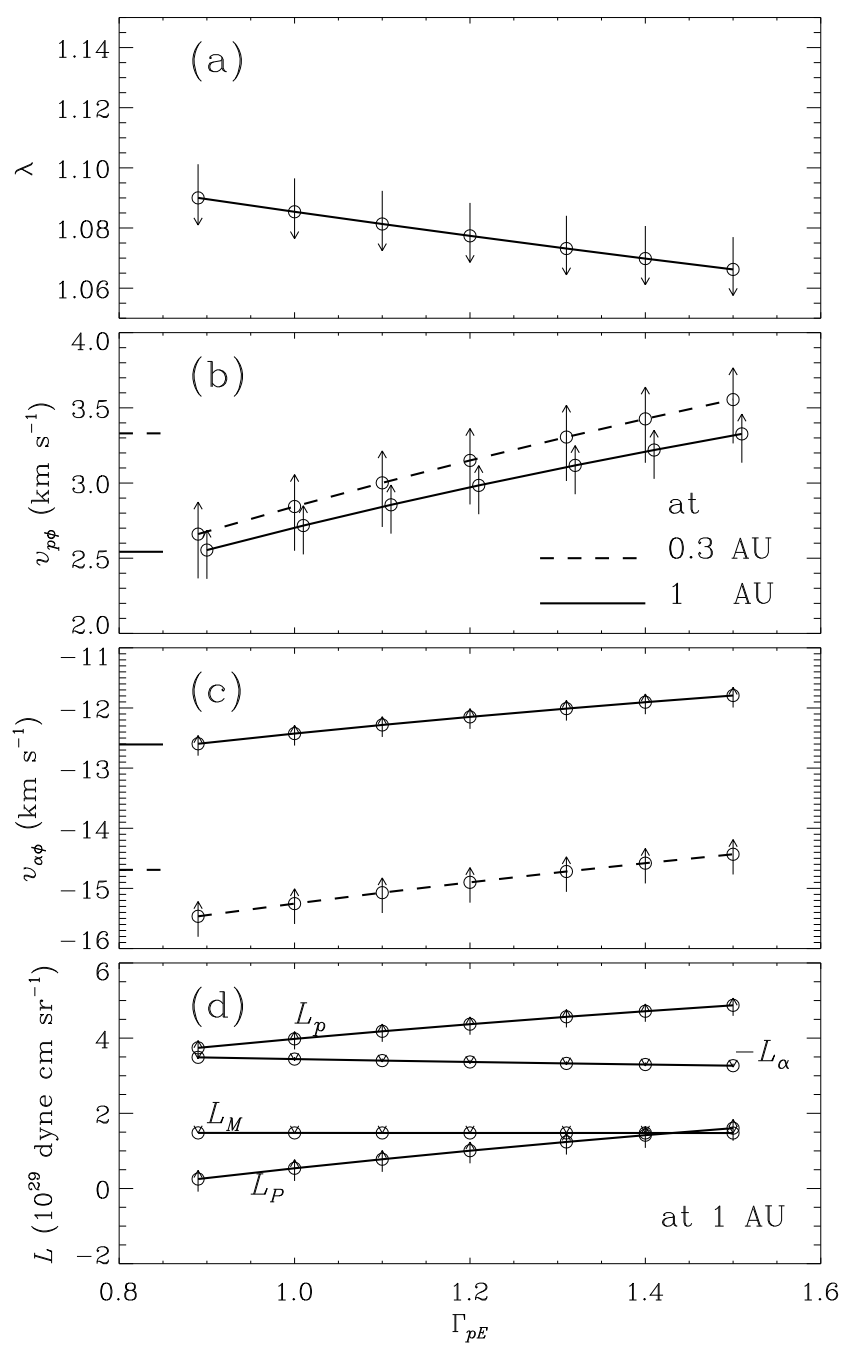

Fig. 4. Values of several parameters as a function of $\Gamma_{\mathrm{pE}}$, the proton temperature anisotropy at $1 \mathrm{AU}$. a) The factor $\lambda$, the deviation of which from unity represents the correction to the total angular momentum loss due to the introduction of ion pressure anisotropies; $\mathbf{b}$ ) and $\mathbf{c}$ ) the proton and alpha azimuthal speeds $v_{\mathrm{p} \phi}$ and $v_{\alpha \phi}$ at two different heliocentric distances given by different line styles as indicated in b); d) various components in the angular momentum flux at $1 \mathrm{AU}$ including individual ion contribution $L_{\mathrm{p}}$ and $L_{\alpha}$, the overall particle contribution $L_{P}=L_{\mathrm{p}}+L_{\alpha}$, as well as the contribution from magnetic stresses $L_{\mathrm{M}}$. Note that $-L_{\alpha}$ instead of $L_{\alpha}$ is given in d). The short horizontal bars in panels b) and c) represent the azimuthal ion speeds derived in the isotropic model for comparison. Furthermore, in panel b) the curve corresponding to $1 \mathrm{AU}$ is slightly shifted relative to that for $0.3 \mathrm{AU}$ to avoid the two overlapping each other. The open circles correspond to the cases where $\Gamma_{\alpha \mathrm{E}}$ is fixed at 1.3 , where $\Gamma_{\alpha \mathrm{E}}$ is the alpha temperature anisotropy at $1 \mathrm{AU}$. At a given $\Gamma_{\mathrm{pE}}$ each parameter varies monotonically from the value with $\Gamma_{\alpha \mathrm{E}}=0.7$, represented by the end of the arrow, to the value with $\Gamma_{\alpha \mathrm{E}}=1.9$ given by the arrow head. The ranges in which $\Gamma_{\mathrm{pE}}$ and $\Gamma_{\alpha \mathrm{E}}$ vary are determined from the Helios measurements (see text for details).

solar wind solutions. However, some quantitative differences exist nonetheless. For instance, when $\Gamma_{\mathrm{pE}}$ is held fixed, all the examined parameters for the slow wind vary little even though $\Gamma_{\alpha \mathrm{E}}$ changes considerably from 0.8 to 2 . In contrast, the parameters for the fast wind show an obvious $\Gamma_{\alpha \mathrm{E}}$ dependence. This difference can be largely attributed to the fact that in the slow wind the ions are substantially cooler than in the fast wind. Figure 5a shows that $\lambda$ ranges from 0.94 to 1.016 . In other words, relative to the isotropic case, the solar angular momentum loss rate

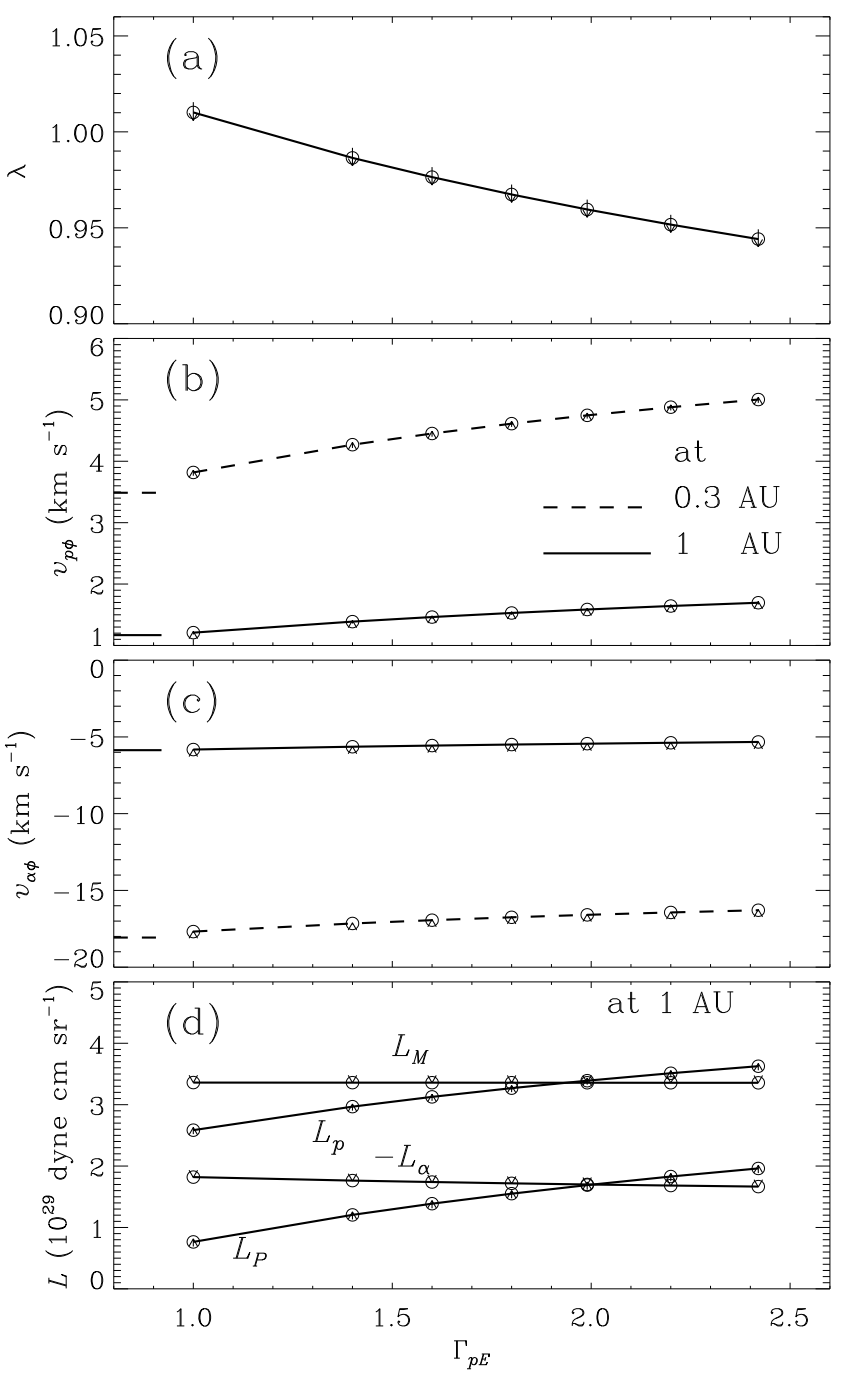

Fig. 5. Similar to Fig. 4 but for the slow solar wind. Here the open circles correspond to the cases where $\Gamma_{\alpha \mathrm{E}}$ is fixed at 1.4, and the arrow represents how the specific parameter varies at a given $\Gamma_{\mathrm{pE}}$ when $\Gamma_{\alpha \mathrm{E}}$ increases from 0.8 to 2.0 .

per steradian in the anisotropic models may be enhanced or reduced by up to $6 \%$. If examining Figs. $5 \mathrm{~b}$ and c, one may find that at both 0.3 and $1 \mathrm{AU}$, the azimuthal speeds of both ion species, $v_{\mathrm{p} \phi}$ and $v_{\alpha \phi}$, are larger algebraically in the anisotropic models than in the isotropic one. The difference between the two is more prominent at $0.3 \mathrm{AU}$, where the isotropic model yields that $\left[v_{\mathrm{p} \phi}, v_{\alpha \phi}\right]=[3.49,-18.1] \mathrm{km} \mathrm{s}^{-1}$, whereas the anisotropic models yield that with increasing $\Gamma_{\mathrm{pE}}, v_{\mathrm{p} \phi}$ increases from 3.76 to $5.04 \mathrm{~km} \mathrm{~s}^{-1}$, and $v_{\alpha \phi}$ varies between -17.8 to $-16.3 \mathrm{~km} \mathrm{~s}^{-1}$. As for the ion azimuthal speeds at $1 \mathrm{AU}$, one can see that varying $\Gamma_{\mathrm{pE}}$ leads to a $v_{\mathrm{p} \phi}$ varying between 1.18 and $1.72 \mathrm{~km} \mathrm{~s}^{-1}$, and a $v_{\alpha \phi}$ ranging from -5.85 to $-5.31 \mathrm{~km} \mathrm{~s}^{-1}$. The corresponding changes in the specific ion angular momentum fluxes are shown by Fig. $5 d$, which indicates that the proton one $L_{\mathrm{p}}$ increases with increasing $\Gamma_{\mathrm{pE}}$ from 2.52 to 3.67 , and likewise, the alpha one $L_{\alpha}$ increases from -1.83 to -1.66 . On the other hand, the flux associated with magnetic stresses $L_{\mathrm{M}}$ hardly varies, and a value of 3.36 can be quoted for all the models examined. Therefore in the parameter space explored, $L_{\mathrm{M}}$ may be smaller than $L_{\mathrm{p}}$, which is however offset by the alpha contribution that is always in the direction of counter-rotation to the Sun. In fact, the alpha contribution is so significant that the overall particle contribution $L_{P}$ 


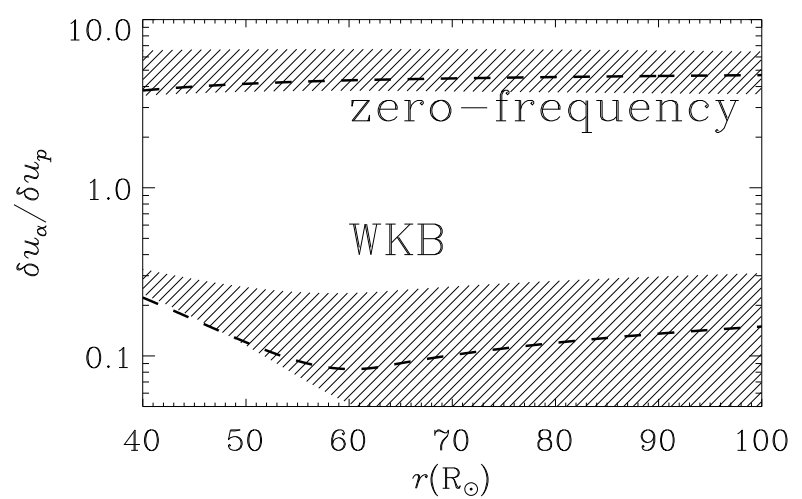

Fig. 6. Radial dependence of the ratio of the alpha to the proton velocity fluctuation amplitudes $\delta u_{\alpha} / \delta u_{\mathrm{p}}$ induced by Alfvénic activities in super-Alfvénic portions of the fast solar wind. The dashed curves correspond to the isotropic model, while the hatched areas give the possible range $\delta u_{\alpha} / \delta u_{\mathrm{p}}$ may occupy when the parameters $\Gamma_{\mathrm{pE}}$ and $\Gamma_{\alpha \mathrm{E}}$ vary in the ranges given in text. Both the zero-frequency (upper portion) and WKB (lower) estimates are given.

never exceeds $L_{\mathrm{M}}$. In other words, incorporating ion temperature anisotropy cannot resolve the outstanding discrepancy between previous models and observations concerning the relative importance of particle and magnetic contributions in the angular momentum budget of the solar wind.

\section{Discussion}

As demonstrated by Li \& Li (2008), the discussion on the angular momentum transport also allows us to say a few words on the frequency spectra $S_{k}(f)(k=\mathrm{p}, \alpha)$ of the ion velocity fluctuations during Alfvénic activities in the fast solar wind in the super-Alfvénic portion where $M_{\mathrm{T}}^{2} \gg 1$. This is due to the well-known change of the properties of Alfvénic fluctuations around some $f_{\mathrm{c}} \approx v_{\mathrm{cm}, \mathrm{a}} /\left(4 \pi r_{\mathrm{a}}\right)$, where $v_{\mathrm{cm}, \mathrm{a}}$ is the speed of center of mass evaluated at the Alfvén point $r_{\mathrm{a}}$ (see e.g., Heinemann \& Olbert 1980; Li \& Li 2008). For typical fast wind parameters, $f_{\mathrm{c}} \approx 0.5-1 \times 10^{-5} \mathrm{~s}^{-1}$. While the fluctuations with frequencies $f \gtrsim f_{\mathrm{c}}$ are genuinely wave-like and may be described by the WKB limit given the slow spatial variation of flow parameters in the region in question, those with $f \lesssim f_{\mathrm{c}}$ behave in a quasi-static manner and may be described by the solutions to the angular momentum conservation law which also governs the zero-frequency fluctuations. As shown by Li \& Li (2008) who neglected the species temperature anisotropy, in the region $r \gtrsim 0.2 \mathrm{AU}$ which will be explored by the Solar Orbiter and Solar Probe, the ratio of the alpha to proton velocity fluctuation amplitude $\delta u_{\alpha} / \delta u_{\mathrm{p}}$ can be an order-of-magnitude larger for $f<f_{\mathrm{c}}$ than for $f>f_{\mathrm{c}}$. Hence one may expect that, if the proton velocity fluctuation spectrum $S_{\mathrm{p}}(f)$ is somehow smooth around $f_{\mathrm{c}}$, then the alpha one $S_{\alpha}(f)$ will show an apparent spectral break. Now let us revisit this problem in light of the discussion presented in this paper and see what changes the pressure anisotropies may introduce.

Restrict ourselves to either the high-latitude region or the region inside say $100 R_{\odot}$ such that the magnetic field may be seen as radial. Furthermore, suppose that the waves are propagating parallel to the magnetic field in the empirical fast wind profiles detailed in Sect. 3. Figure 6 presents the radial dependence of $\delta u_{\alpha} / \delta u_{\mathrm{p}}$ in the region between 40 and $100 R_{\odot}$ for both the zero-frequency (upper part) and WKB (lower part) solutions. For comparison, the dashed curves represent the corresponding results in the isotropic model. To construct Fig. 6, all the possible values of $\Gamma_{\mathrm{pE}}$ and $\Gamma_{\alpha \mathrm{E}}$ have been examined. As a result, at any radial location the ratio $\delta u_{\alpha} / \delta u_{\mathrm{p}}$ varies from model to model, and the range in which this ratio may occupy is given by the hatched area. The zero-frequency solutions are obtained by solving Eq. (20), while for hydromagnetic WKB Alfvén waves it is well known that $\delta u_{\alpha} / \delta u_{\mathrm{p}}=\left|\left(v_{\mathrm{ph}}-v_{\alpha}\right) /\left(v_{\mathrm{ph}}-v_{\mathrm{p}}\right)\right|$, where $v_{\mathrm{ph}}$ is the wave phase speed and given by (e.g., Barnes \& Suffolk 1971; Isenberg 1984)

$v_{\mathrm{ph}}=v_{\mathrm{cm}}+\sqrt{v_{\mathrm{A}}^{2}\left(1-\frac{4 \pi P^{\Delta}}{B^{2}}\right)-\hat{\rho}_{\alpha} \hat{\rho}_{\mathrm{p}} v_{\alpha \mathrm{p}}^{2}}$,

in which $v_{\mathrm{cm}}=\hat{\rho}_{\mathrm{p}} v_{\mathrm{p}}+\hat{\rho}_{\alpha} v_{\alpha}$ is the speed of center of mass, and $\hat{\rho}_{k}=\rho_{k} / \rho(k=\mathrm{p}, \alpha)$ defines the fractional ion mass density.

From Fig. 6 one can see that the zero-frequency and WKB solutions are well separated from each other, in the isotropic and anisotropic cases alike. For the isotropic model, $\delta u_{\alpha} / \delta u_{\mathrm{p}}$ in the zero-frequency case increases monotonically from 3.79 at $40 R_{\odot}$ to 4.68 at $100 R_{\odot}$. On the other hand, in the WKB case it decreases first from 0.22 at $40 R_{\odot}$ and attains its minimum of 0.083 at $60.3 R_{\odot}$ and then increases to 0.15 at $100 R_{\odot}$. The difference in $\delta u_{\alpha} / \delta u_{\mathrm{p}}$ between the zero-frequency and WKB solutions may be slightly smaller in the anisotropic than in the isotropic case for some combinations of $\left[\Gamma_{\mathrm{pE}}, \Gamma_{\alpha \mathrm{E}}\right]$, but the difference is still quite significant. From this we can conclude that, with realistic ion temperature anisotropies included, the alpha velocity fluctuation spectrum $S_{\alpha}(f)$ during Alfvénic activities will also show an apparent break near $f_{\mathrm{c}}$, if the proton one $S_{\mathrm{p}}(f)$ is smooth there. This break is entirely a linear property, and has nothing to do with the nonlinearities that may also shape the fluctuation spectra.

\section{Summary}

This study has been motivated by the apparent lack of an analysis on the angular momentum transport in a multicomponent solar or stellar wind with differentially flowing ions and species temperature anisotropy. Moreover, there has been an outstanding discrepancy between available measurements and models concerning the relative importance of the particle $L_{P}$ and magnetic contribution $L_{\mathrm{M}}$ to the solar angular momentum loss rate per steradian $L$. The Helios measurements indicate that for fast (slow) solar wind with $v_{\mathrm{p}} \gtrsim 600(\$ 400) \mathrm{km} \mathrm{s}^{-1}, L_{P}$ tends to be negative (positive), with the positive sign denoting the direction of corotation with the Sun. Furthermore, $L_{P}$ tends to be larger than $L_{\mathrm{M}}$ in the slow wind. The behavior of $L_{P}$ derives from that of individual ion angular momentum fluxes, $L_{\mathrm{p}}$ and $L_{\alpha}$, thereby calling for a multifluid approach.

Starting with a general set of multifluid transport equations with gyrotropic species pressure tensors, we have derived the equations for both the angular momentum conservation (Eqs. (10) and (20) in Sect. 2), and the energy and linear momentum balance (Eqs. (A.1) to (A.4) in the Appendix). These equations are not restricted to radial outflows in the equatorial plane, instead they are valid for arbitrary axisymmetrical winds that include two major ion species, and therefore are expected to find applications in general outflows from late-type stars. To focus on the problem of angular momentum transport, we refrained from solving the full set of equations governing the meridional dynamics. Rather, we constructed, largely based on the available in situ measurements, the empirical profiles for the meridional magnetic field and flow parameters. Only the ion temperature anisotropies are considered, i.e., the electron temperature is 
seen as isotropic. For both the fast and slow solar wind profiles, we solved the angular momentum conservation law (Eqs. (10) and (20)) to examine how the azimuthal speeds of protons $v_{\mathrm{p} \phi}$ and alpha particles $v_{\alpha \phi}$, as well as the individual components in the solar angular momentum budget are influenced by the ion temperature anisotropies. To this end, solutions to the isotropic version are obtained for comparison.

Our main conclusions are:

1. From the derived equations governing the energy transport, a simple analysis given in the Appendix yields that the adiabatic cooling may be considerably influenced with the introduction of the azimuthal components. Such an influence is understandably more prominent in the low-latitude regions. This means, when modeling the species temperature anisotropy, for a quantitative comparison of model computations to be made with the near-ecliptic measurements such as made by Helios, the spiral magnetic field has to be taken into account.

2. In agreement with the single-fluid case (Weber \& Davis 1970; Weber 1970), incorporating species temperature anisotropy leads to a situation where the total angular momentum loss rate per steradian $L$ is determined by the behavior of the solution to the angular momentum conservation law in the vicinity of the Alfvén point where the combined Alfvénic Mach number $M_{\mathrm{T}}=1$. However, $M_{\mathrm{T}}$ has to take into account the contribution from both ion species, as defined by Eq. (18).

3. Relative to the isotropic case, the introduced species temperature anisotropy may enhance or decrease $L$ by up to $10 \%$, and introduce an absolute change of up to $\sim 1.8 \mathrm{~km} \mathrm{~s}^{-1}$ in individual ion azimuthal speeds in the region between 0.3 and 1 AU. While these changes seem modest, the corresponding changes in the angular momentum fluxes convected by protons $L_{\mathrm{p}}$ or alpha particles $L_{\alpha}$ may change substantially. In contrast, the flux associated with magnetic stresses $L_{\mathrm{M}}$ hardly varies.

4. However, introducing ion temperature anisotropies cannot resolve the discrepancy between in situ measurements and models. For the fast wind solutions, while in extreme cases $L_{P}$ may become negative $L_{\mathrm{p}}$ always stays positive. On the other hand, for the slow solar wind solutions examined, $L_{P}$ never exceeds $L_{\mathrm{M}}$ even though $L_{\mathrm{M}}$ may be smaller than the individual ion contribution. This is because, for both the slow and fast wind solutions, $L_{\mathrm{p}}$ and $L_{\alpha}$ always have opposite signs.

5. The discussion on the angular momentum transport has some bearing on the ion velocity fluctuation spectra $S_{k}(f)(k=$ $\mathrm{p}, \alpha)$ during Alfvénic activities in the super-Alfvénic regions, which are likely to be explored by future missions such as Solar Orbiter and Solar Probe. In agreement with Li \& Li (2008) where species temperature anisotropies are neglected, an analysis based on the WKB and zero-frequency solutions yields that $S_{\alpha}(f)$ will show an apparent break around some critical frequency $f_{\mathrm{c}}$ if $S_{\mathrm{p}}(f)$ is smooth there. This $f_{\mathrm{c}} \sim 0.5-1 \times 10^{-5} \mathrm{~s}^{-1}$ is the well-known frequency that separates the genuinely wave-like fluctuations from quasi-static ones.

Acknowledgements. We thank the referee (Dr. Horst Fichtner) for his very helpful comments. This research is supported by an STFC rolling grant to Aberystwyth University.

\section{Appendix A: Derivation of equations governing the meridional dynamics}

In Sect. 2, we have demonstrated that the vector equations governing a time-independent multicomponent solar wind with species temperature anisotropy are allowed to be decomposed into a force balance condition across the poloidal magnetic field and a set of transport equations along it. The azimuthal dynamics has been discussed in the text, whereas this Appendix provides some discussion on the poloidal dynamics. In particular, we shall derive the equations governing the poloidal motion $v_{k l}$ of ion species $(k=\mathrm{p}, \alpha)$, and the species temperatures $T_{s}^{\|, \perp}(s=\mathrm{e}, \mathrm{p}, \alpha)$ in rather general situations.

Due to the presence of $v_{k N}$ in the $l$ component of the ion momentum Eq. (2), one may expect that the $N$-component of Eq. (2) has to be solved. In fact, there is no need to do so because $v_{k N}$ appears only in the difference $v_{j N}-v_{k N}$, which may be found from the $\phi$ component of Eq. (2). Substituting $v_{j N}-v_{k N}$ into the $l$ component of Eq. (2) will then eliminate the cumbersome $\Omega_{k}$ and $v_{k N}$. Note that this technique, first devised by McKenzie et al. (1979), ensures the conservation of not only total momentum but also total energy (see Li \& Li 2006). Specifically, the resulting equations for the poloidal dynamics are

$$
\begin{aligned}
\left(\frac{n_{k} v_{k l}}{B_{l}}\right)^{\prime}= & 0 \\
v_{k l}\left(v_{k l}\right)^{\prime}- & v_{k \phi}^{2}(\ln R)^{\prime}+\tan \Phi \frac{v_{k l}}{R}\left(R v_{k \phi}\right)^{\prime} \\
& -\left(C_{k l}+\tan \Phi C_{k \phi}\right)+\frac{G M_{\odot}}{r}(\ln r)^{\prime} \\
& +\frac{1}{n_{k} m_{k}}\left\{\left(p_{k}^{\|}\right)^{\prime}-p_{k}^{\Delta}\left[\ln \left(B_{l} \sec \Phi\right)\right]^{\prime}\right\} \\
& +\frac{Z_{k}}{n_{\mathrm{e}} m_{k}}\left\{\left(p_{\mathrm{e}}^{\|}\right)^{\prime}-p_{\mathrm{e}}^{\Delta}\left[\ln \left(B_{l} \sec \Phi\right)\right]^{\prime}\right\}=0 \\
v_{s l}\left(T_{s}^{\|}\right)^{\prime}+ & 2 v_{s l} T_{s}^{\|}\left[\ln \left(v_{s l} \sec \Phi\right)\right]^{\prime} \\
& +\frac{1}{n_{s} k_{\mathrm{B}}}\left[\nabla \cdot \boldsymbol{q}_{s}^{\|}-\mathrm{Q}_{s}: \nabla(\hat{b} \hat{b})-\frac{\delta E_{s}^{\|}}{\delta t}-H_{s}^{\|}\right]=0 \\
v_{s l}\left(T_{s}^{\perp}\right)^{\prime}- & v_{s l} T_{s}^{\perp}\left[\ln \left(B_{l} \sec \Phi\right)\right]^{\prime} \\
& +\frac{1}{n_{s} k_{\mathrm{B}}}\left[\nabla \cdot \boldsymbol{q}_{s}^{\perp}+\frac{\mathrm{Q}_{s}}{2}: \nabla(\hat{b} \hat{b})-\frac{\delta E_{s}^{\perp}}{\delta t}-H_{s}^{\perp}\right]=0
\end{aligned}
$$

where $p_{s}^{\Delta}=p_{s}^{\|}-p_{s}^{\perp}(s=\mathrm{e}, \mathrm{p}, \alpha), \boldsymbol{C}_{k}=\delta \boldsymbol{M}_{k} / \delta t+$ $\left(Z_{k} n_{k} / n_{\mathrm{e}}\right) \delta \boldsymbol{M}_{\mathrm{e}} / \delta t$ results from Coulomb frictions, and the prime' represents the derivative with respect to the arclength $l$. When deriving Eqs. (A.3) and (A.4), we have used the fact that the expression

$$
\begin{aligned}
\nabla_{\|} \cdot \boldsymbol{v}_{s}= & \cos ^{2} \Phi v_{s l}^{\prime}+\sin ^{2} \Phi v_{s l}(\ln R)^{\prime} \\
& +\cos \Phi \sin \Phi R\left(v_{s \phi} / R\right)^{\prime}
\end{aligned}
$$

may be simplified by expressing $v_{s \phi}$ via the alignment condition (10), the result being

$\nabla_{\|} \cdot \boldsymbol{v}_{s}=v_{s l}\left[\ln \left(v_{s l} \sec \Phi\right)\right]^{\prime}$

Similarly, one may find that

$\nabla_{\perp} \cdot \boldsymbol{v}_{s}=-v_{s l}\left[\ln \left(B_{l} \sec \Phi\right)\right]^{\prime}$.

Now let us compare these equations with those in Isenberg (1984). It is straightforward to show that Eqs. (A.3) and (A.4) are equivalent to Eqs. (A.3) and (A.4) in Isenberg (1984) by specializing to a spherically symmetric solar wind and by noting 
that $\left(\ln B_{l}\right)^{\prime}=-2 / r$. On the other hand, using the alignment condition (10) one may find that

$$
\begin{array}{r}
v_{k l}\left(v_{k l}\right)^{\prime}-v_{k \phi}^{2}(\ln R)^{\prime}+\tan \Phi \frac{v_{k l}}{R}\left(R v_{k \phi}\right)^{\prime}= \\
\left(\frac{v_{k l}^{2}}{2} \sec ^{2} \Phi\right)^{\prime}-\left(\frac{A_{\Omega}^{2} R^{2}}{2}\right)^{\prime} .
\end{array}
$$

Again specializing to a spherical solar wind, one then finds that Eq. (A.2) is equivalent to (A.2) in Isenberg (1984). It should be stressed that although working in a frame of reference corotating with the Sun, as did Isenberg (1984), substantially simplifies the algebra, it does not offer the information on the specific form of the spiral angle $\Phi$, whose functional dependence on the flow speeds has to be assumed a priori. In practice, Isenberg (1984) assumed that the velocity of center of mass $\boldsymbol{v}_{\mathrm{cm}}$ is radial in an inertial frame beyond $10 R_{\odot}$, which is certainly a good assumption for the present slow-rotating Sun. However, from our discussion on the azimuthal dynamics, there is in general no guarantee that $\boldsymbol{v}_{\mathrm{cm}}$ is radial, and the deviation may be substantial for winds that flow from a faster rotating star.

Introducing azimuthal components may influence the ion flow speeds $v_{k l}$ both directly and indirectly. The direct consequence is that azimuthal components may introduce into the reduced meridional momentum Eq. (A.2) an effective force (see the first three terms). Note that in a corotating frame the magnitude of the ion velocity becomes $v_{k l} \sec \Phi$, from relation (A.5) one may see that in such a frame all particles move in the same centrifugal potential $A_{\Omega}^{2} R^{2} / 2$. Therefore in effect the introduced force tends to reduce the magnitude of the ion speed difference with increasing distance as $\sec \Phi$ tends to increase. This effect has been explored in detail in Li \& Li (2006) and Li et al. (2007), where it is shown that the influence may play an important part in the force balance for the solar wind. In fact, introducing solar rotation alone is able to reproduce the $v_{\alpha p}$ profile measured by Ulysses beyond $2 \mathrm{AU}$ if a proper value of $v_{\alpha \mathrm{p}}$ is imposed there. On the other hand, $v_{k l}$ may be altered indirectly by the modified pressure gradient force due to changes in the temperatures, which in turn are caused by the changes in the heat fluxes (the third term in Eqs. (A.3) and (A.4)) and through the adiabatic cooling (the second term). A detailed discussion on the former requires a specific form for the heat flux, which is beyond the scope of the present paper. As a consequence, we shall focus on the latter instead.

Neglecting the terms in the second pair of square parentheses, Eqs. (A.3) and (A.4) give

$T_{s}^{\|} \propto \cos ^{2} \Phi / v_{s l}^{2}, T_{s}^{\perp} \propto B_{l} \sec \Phi$.

Note that the relation governing $T_{s}^{\perp}$ simply reflects the conservation of magnetic moment. Now that in the region say $r>10 R_{\odot} \sec \Phi$ is significant and increases with $r, T_{s}^{\|}\left(T_{s}^{\perp}\right)$ may be substantially reduced (enhanced) relative to the case where $\Phi \equiv 0$. This effect is particularly significant in the near-ecliptic region and for the slow solar wind. For instance, restrict ourselves to the equatorial plane and consider the region between say $10 R_{\odot}$ and $1 \mathrm{AU}$. Suppose $v_{s l}$ remains constant and $v_{s l} \approx A_{\Omega} R_{\mathrm{E}}=430 \mathrm{~km} \mathrm{~s}^{-1}$. Now that roughly speaking $\tan \Phi \approx-A_{\Omega} r / v_{s l}$, when the spiral field is considered, $T_{s}^{\|}\left(T_{s}^{\perp}\right)$ at $1 \mathrm{AU}$ is $1 / 2(\sqrt{2})$ times the value for a purely radial magnetic field. This suggests that for making any quantitative comparison of the modeled species temperature anisotropy with the near-ecliptic measurements such as made by Helios, the spiral magnetic field has to be considered.

For completeness, we note that the force balance condition across the $N$ direction comes from the $N$ component of the total momentum, which reads

$$
\begin{aligned}
\sum_{k} \rho_{k}\left(\frac{v_{k l}^{2}}{R_{\mathrm{c}}}\right. & \left.-v_{k \phi}^{2} \frac{\partial}{\partial N} \ln R\right)+\frac{\partial}{\partial N}\left(P^{\perp}+\frac{B^{2}}{8 \pi}\right) \\
& -\frac{1}{4 \pi}\left(1-\frac{4 \pi P^{\Delta}}{B^{2}}\right)\left(\frac{B_{l}^{2}}{R_{\mathrm{c}}}-B_{\phi}^{2} \frac{\partial}{\partial N} \ln R\right) \\
& +\sum_{k} \rho_{k} \frac{G M_{\odot}}{r} \frac{\partial}{\partial N} \ln r=0,
\end{aligned}
$$

where $R_{\mathrm{c}}=\hat{e}_{N} \cdot\left(\hat{e}_{l} \cdot \nabla \hat{e}_{l}\right)$ is the signed curvature radius of the poloidal magnetic line of force. Obviously, this force balance condition determines the poloidal magnetic field configuration in response to the electric currents associated with the flow. This equation, combined with the transport equations along the meridional magnetic lines of force, may be solved alternately to find a self-consistent solution to the vector equations by using the approach by Pneuman \& Kopp (1971) or Sakurai (1985).

\section{References}

Banaszkiewicz, M., Axford, W. I., \& McKenzie, J. F. 1998, A\&A, 337, 940 Barakat, A. R., \& Schunk, R. W. 1982, Plasma Phys., 24, 389 Barnes, A., \& Suffolk, G. C. J. 1971, J. Plasma Phys., 5, 315 Belcher, J. W., \& MacGregor, K. B. 1976, ApJ, 210, 498 Bouvier, J., Forestini, M., \& Allain, S. 1997, A\&A, 326, 1023 Frazin, R. A., Cranmer, S. R., \& Kohl, J. L. 2003, ApJ, 597, 1145

Gary, S. P., McKean, M. E., Winske, D., et al. 1994, J. Geophys. Res., 99, 5903 Heinemann, M., \& Olbert, S. 1980, J. Geophys. Res., 85, 1311 Hellinger, P., \& Trávníček, P. 2006, J. Geophys. Res., 111, A01107 Hollweg, J. V., \& Isenberg, P. A. 2002, J. Geophys. Res., 107(A7), 1147 Hu, Y. Q., \& Habbal, S. R. 1999, J. Geophys. Res., 104, 17045 Isernberg, P. A. 1984, J. Geophys. Res., 89, 6613

Kohl, J. L., Noci, G., Antonucci, E., et al. 1998, ApJ, 501, L127

Leer, E., \& Axford, W. I. 1972, Sol. Phys., 23, 238

Li, B., \& Li, X. 2006, A\&A, 456, 359

Li, B., \& Li, X. 2007, ApJ, 661, 1222

Li, B., \& Li, X. 2008, ApJ, 682, 667

Li, B., Li, X., \& Labrosse, N. 2006, J. Geophys. Res., 111, A08106

Li, B., Habbal, S. R., \& Li, X. 2007, ApJ, 661, 593

Marsch, E., \& Richter, A. K. 1984, J. Geophys. Res., 89, 5386

Marsch, E., Mühlhäuser, K.-H., Rosenbauer, H., Schwenn, R., \& Denskat, K. U. 1981, J. Geophys. Res., 86, 9199

Marsch, E., Mühlhäuser, K.-H., Rosenbauer, H., Schwenn, R., \& Neubauer, F. M. 1982a, J. Geophys. Res., 87, 35 (M82a)

Marsch, E., Mühlhäuser, K.-H., Schwenn, R., et al. 1982b, J. Geophys. Res., 87, 52 (M82b)

McComas, D. J., Barraclough, B. L., Funsten, H. O., et al. 2000, J. Geophys. Res., 105, 10419 (Mc00)

McKenzie, J. F., Ip, W.-H., \& Axford, W. I. 1979, Ap\&SS, 64, 183

Mestel, L., \& Spruit, H. C. 1987, MNRAS, 226, 57

Pizzo, V., Schwenn, R., Marsch, E., et al. 1983, ApJ, 271, 335

Pneuman, G. W., \& Kopp, R. A. 1971, Sol. Phys., 18, 258

Sakurai, T. 1985, A\&A, 152, 121

Scherer, K., Marsch, E., Schwenn, R., \& Rosenbauer, H. 2001, A\&A, 366, 331

Schwenn, R. 1990, Large-scale structure of the interplanetary medium, in Physics of the Inner Heliosphere I, Large Scale Phenomena, ed. R. Schwenn, \& E. Marsch (New York: Springer-Verlag), 99 (Sch90)

Smith, E. J., \& Balogh, A. 1995, Geophys. Res. Lett., 22, 3317

Weber, E. J. 1970, Sol. Phys., 13, 240

Weber, E. J., \& Davis, Jr., L. 1967, ApJ, 148, 217

Weber, E. J., \& Davis, Jr., L. 1970, J. Geophys. Res., 75, 2419 